\title{
Improved ascertainment of modes of HIV transmission in Ukraine indicates importance of drug injecting and homosexual risk
}

Kostyantyn Dumchev ${ }^{1 *}$ (D) Marina Kornilova ${ }^{2}$, Roksolana Kulchynska ${ }^{3}$, Marianna Azarskova ${ }^{3}$ and Charles Vitek ${ }^{4}$

\begin{abstract}
Background: It is important to understand how HIV infection is transmitted in the population in order to guide prevention activities and properly allocate limited resources. In Ukraine and other countries where injecting drug use and homosexuality are stigmatized, the information about mode of transmission in case registration systems is often biased.

Methods: We conducted a cross-sectional survey in a random sample of patients registered at HIV clinics in seven regions of Ukraine in 2013-2015. The survey assessed behavioral risk factors and serological markers of viral hepatitis B and C. We analyzed the discrepancies between the registered mode of transmission and the survey data, and evaluated trends over 3 years.

Results: Of 2285 participants, 1032 (45.2\%) were females. The proportion of new HIV cases likely caused by injecting drug use based on the survey data was $59.7 \%$ compared to $33.2 \%$ in official reporting, and proportion of cases likely acquired through homosexual transmission was $3.8 \%$ compared to $2.8 \%$. We found a significant decrease from 63.2 to $57.5 \%$ in the proportion of injecting drug use-related cases and a steep increase from 2.5 to $5.2 \%$ in homosexual transmission over 3 years.

Conclusions: The study confirmed the significant degree of misclassification of HIV mode of transmission among registered cases. The role of injecting drug use in HIV transmission is gradually decreasing, but remains high. The proportion of cases related to homosexual transmission is relatively modest, but is rapidly increasing, especially in younger men. Improvements in ascertaining the risk factor information are essential to monitor the epidemic and to guide programmatic response.
\end{abstract}

Keywords: HIV epidemiology, HIV transmission, HIV risk factors, Injecting drug use, Ukraine

\footnotetext{
*Correspondence: dumchev@uiphp.org.ua

${ }^{1}$ Ukrainian Institute on Public Health Policy, Kyiv, Ukraine

Full list of author information is available at the end of the article
}

(c) The Author(s). 2020 Open Access This article is licensed under a Creative Commons Attribution 4.0 International License, which permits use, sharing, adaptation, distribution and reproduction in any medium or format, as long as you give appropriate credit to the original author(s) and the source, provide a link to the Creative Commons licence, and indicate if changes were made. The images or other third party material in this article are included in the article's Creative Commons licence, unless indicated otherwise in a credit line to the material. If material is not included in the article's Creative Commons licence and your intended use is not permitted by statutory regulation or exceeds the permitted use, you will need to obtain permission directly from the copyright holder. To view a copy of this licence, visit http://creativecommons.org/licenses/by/4.0/. The Creative Commons Public Domain Dedication waiver (http://creativecommons.org/publicdomain/zero/1.0/) applies to the data made available in this article, unless otherwise stated in a credit line to the data. 


\section{Background}

The "Know your HIV epidemic" approach introduced and guided by the Joint United Nations Programme on HIV/AIDS has become a critical part of the HIV/AIDS response [1]. Understanding trends in HIV transmission in high-risk key populations is crucial for optimal allocation of limited prevention resources.

Available data about modes of HIV transmission are often suboptimal not only because of the stigma associated with HIV risk behaviors but also because of systemic flaws in reporting systems. Most case-reporting systems collect information on modes of HIV transmission based on patients' self-reporting, which is usually neither accurate nor complete because this requires disclosure of sensitive and potentially stigmatizing information [2, 3]. For example, in the US, completion of risk factor information in case report forms submitted to the HIV/AIDS Reporting System is not mandatory, which contributed to $<80 \%$ of cases among men being reported within a known transmission category in 2014 [4]. Despite the relatively low level of stigma in the US, men who have sex with men (MSM) and injecting drug use behaviors were underreported: about 77\% of 6891 men who did not report a transmission category were estimated to be MSM and $8 \%$ to be persons who inject drugs (PWID) [4].

In Ukraine, which has the second largest HIV epidemic in Europe [5], the case registration system captures nearly all cases with confirmed HIV infection, but introduces two potential biases in assessing the mode of transmission. First, the registration form, filled once at the time of diagnosis confirmation, does not include fields for specific risk factors, and does not provide guidelines for structured collection of risk factor data. The substantial stigma toward injecting drug use and MSM [6] may lead to underreporting of these behaviors by the patients and incorrect assumptions by healthcare providers. Second, unlike in the US, where CDC developed a hierarchy of transmission categories [7], Ukraine's system lacks guidance on how to rank multiple risk factors and establish the probable mode of transmission. As a result, the indirect evidence indicates that modes of transmission are substantially misclassified [8]. An analysis of hepatitis $\mathrm{C}$ virus (HCV) prevalence among HIV-positive men in 2009 suggested that as many as $34.5 \%$ of men registered with heterosexual exposure as the mode of transmission could in fact have acquired HIV through injecting drug use [8]. Extrapolation of national biobehavioral survey data suggests that another $8.3 \%$ of these men could be MSM [8,9]. A triangulation study, combining all existing data sources, suggested that HIV transmission remained linked to PWID and their sexual partners [10].

According to official reported case registration data, heterosexual exposure was the dominant mode of HIV transmission in Ukraine in 2008, accounting for $70.1 \%$ of 12,893 adults (aged $\geq 15$ years) registered in 2015 [11]. Injecting drug use, once the main driver of the HIV epidemic, has decreased to $26.8 \%$. The number of cases officially attributed to homosexual exposure increased steadily, from 20 in 2005 to 368 in 2015. These trends and the fact that the absolute number and rate of new HIV infections started decreasing in 2012, following the overall trend in Eastern Europe (excluding Russia) [5], may suggest that injecting drug use is no longer, and that transmission among MSM is not yet, a major factor in the Ukrainian HIV epidemic. Additionally, the high proportion of cases attributed to heterosexual exposure and declining levels of HIV among female sex workers [12] would support directing resources toward prevention of heterosexual transmission in the general population. However, if the magnitude of misclassification of the mode of transmission is as significant as previous evidence suggests, these conclusions would be invalid.

The primary goal of this study was to assess the risk factors among patients diagnosed with HIV using a sensitive algorithm and estimate the degree of misclassification in the registered mode of HIV transmission. This evidence will inform improvement in the official HIV case registration system including the update of the case reporting form and training of healthcare providers involved in assessing modes of transmission. The secondary goal of the study was to assess the changes in the main transmission categories from 2013 to 2015 and determine the epidemic trends.

\section{Methods}

\section{Study design and population}

In Ukraine, individuals who screen positive for HIV at any community- or facility-based testing sites are referred to a network of government HIV clinics for confirmatory testing, diagnosis and registration. To assess the HIV risk factors and biological markers of transmission, we conducted a cross-sectional survey among adult patients who were officially registered at all clinical facilities in three most recent years before the study, from 2013 to 2015.

\section{Sampling}

We used a two-step random sampling approach to recruit participants into the study. At the first step, we selected seven out of 27 administrative regions of Ukraine using a random number generator. To estimate the prevalence of HIV risk factors with a 95\% confidence level, 5\% margin of error and assuming a survey design effect of 2.0, the target sample size was 775 per each year. To ensure representation of regions with different sizes of the epidemic (which could potentially be associated with case registration practices), we sorted the list of regions by the number of HIV cases reported in 2013 [13] and chose each fourth 
unit starting from a randomly generated number. The target sample size was divided across the seven regions proportionally to the number of cases reported in 2013 (Supplement Table S1).

Since there was no significant change in the number of registered patients in the selected regions over time, we chose to sample patients from three equal 3-month periods (October-December) to investigate the change in mode of transmission over time.

At the second step, we used a random sampling approach to recruit survey participants. We extracted data from patient registration forms to create a de-identified registry of patients diagnosed during the specified three periods (Supplement Table S2). The data included personal within-clinic ID code, sex, year of birth, date of registration, date of discharge (if applicable) and reason of discharge, dates of HIV tests used for diagnosis, registered mode of transmission, and stage of HIV disease at the time of diagnosis. In a random order, patients from the registry were contacted and invited to participate in the survey. All patients who attended the study appointment provided written informed consent.

\section{Data collection}

After providing informed consent, patients completed an anonymous survey to determine the patients' HIV risk factors 10 years before finding out about their HIV-positive status. The survey instrument was developed by the investigators specifically for this study, and was pilot tested on 10 patients to adjust wording that could be misinterpreted. The questionnaire (see Supplementary File 1) included the following sections: sociodemographic information, sexual risk factors and protective behaviors, injecting drug use history, and history of sexually transmitted or bloodborne infections. The survey was administered by trained interviewers using the REDCap electronic data capture tools hosted at Ukrainian Institute on Public Health Policy [14]. To limit self-reporting bias, interviewers were trained in psychological techniques to enhance rapport with respondents. Although the interviews took place in the HIV clinics, the interviewers were not clinic staff, and the information collected was not disclosed to any clinic staff. No one from the local research teams except the interviewers had access to the REDCap database. The survey data did not include any personal identifiers and were linked to the patient recruitment logs and registries using a participant ID. Before the interview, the interviewers explained that they did not have access to personal information in the clinical records and that the clinical staff did not have access to the interview data. The interview on average took $15 \mathrm{~min}$ to complete.

After the interview, participants provided a blood sample for hepatitis B virus (HBV) and HCV testing. Samples were centrifuged, and plasma samples were frozen. After recruitment, the frozen samples were shipped to a national viral hepatitis reference lab at the Kyiv City AIDS center. The samples were tested for biomarkers of HBV and HCV infection using the ARCHITECT-i1000SR Immunoassay Analyzer and the following reagent kits: ARCHITECT HBsAg, ARCHITECT Anti-HBs, ARCHIT ECT Anti-HBc, ARCHITECT Anti-HBc IgM and ARCH ITECT anti-HCV. Results were reported both to the study investigators and to regional HIV clinics to inform clinicians and the patients about the results of the tests.

Data were collected between March and October 2016.

\section{Data analysis}

The descriptive analysis focused on the distribution of the registered mode of transmission in the official reports and verified registry and on the distribution of HIV risk factors among the survey participants. The data were disaggregated by sex, year of registration, and region. HIV surveillance data represents a census of HIV diagnoses [15] for the selected regions; therefore, no confidence intervals are presented.

In clinical records and official reports, registered modes of transmission were heterosexual exposure, homosexual exposure, injecting drug use, blood product transfusion, transplantation of organs or tissues, other medical exposure, occupational exposure, other nonmedical exposure, confirmed mother-to-child, unconfirmed mother-to-child, and unknown. Mother-to-child transmission cases were excluded from this study. We combined all categories with few cases (except heterosexual exposure, homosexual exposure, and injecting drug use) into an "other" category.

For each risk factor identified in the survey, we created a dichotomous variable based on one or more questions. Some participants were not consistent in responding to different questions addressing the same risk factor; therefore, we constructed logical formulas defining absence or presence of the factor (Table 1). Both in descriptive analysis and in hypothesis testing, we treated these variables as not mutually exclusive, recognizing that one person may be exposed to more than one factor at the same time.

We constructed a summary variable representing the most probable mode of transmission based on the survey responses. Given the strong correlation between $\mathrm{HCV}$ and injecting drug use, presence of anti-HCV antibodies was considered a marker of injecting drug use-related transmission. If no anti-HCV antibodies were detected, the survey-based mode of transmission was based on selfreported behavior. If only one risk factor was reported by a participant, the survey-based mode of transmission was assigned the corresponding value. If a participant reported multiple exposures, the survey-based modes of transmission took the value of a risk factor associated with greater probability of transmission per act [16] and higher level of 
Table 1 Logical formulas for risk factor definitions

\begin{tabular}{|c|c|}
\hline Heterosexual & $\begin{array}{l}\text { heterosexual exposure (regardless of having homosexual exposure) }{ }^{a} \text { OR having one or more partners of the opposite sex } \\
\text { OR self-reporting being infected through heterosexual exposure }\end{array}$ \\
\hline High-risk heterosexual & $\begin{array}{l}\text { having heterosexual exposure (defined above) AND [having had a sexual partner who injects drugs }{ }^{\mathrm{a}} \text { OR having had } \\
\text { heterosexual contact with an HIV-positive person }{ }^{\mathrm{a}} \text { OR giving or receiving money or drugs for sex } \text { en }^{\mathrm{a}} \text { ORaving had a sexual } \\
\text { partner who was bisexual }{ }^{\mathrm{a}} \text { AND being female]] }\end{array}$ \\
\hline Injecting drug use & injecting illicit drugs at least once ${ }^{a}$ OR self-reporting injecting drug use as the most likely mode of HIV transmission \\
\hline Homosexual & $\begin{array}{l}\text { being male AND [having sexual contact with men at the present time OR having one or more male sexual partners }{ }^{\mathrm{a}} \text { OR } \\
\text { having a sexual partner of the same sex }{ }^{\mathrm{a}} \text { OR having homosexual contact with an HIV-positive person }{ }^{\mathrm{a}} \mathrm{OR} \text { self-reporting } \\
\text { homosexual exposure as the most likely mode of HIV transmission] }\end{array}$ \\
\hline Nosocomial & $\begin{array}{l}\text { having had blood or blood product transfusion }{ }^{a} \text { OR having had organ or tissue transplantation }{ }^{a} \text { OR having had in vitro } \\
\text { fertilization }{ }^{a} \text { OR self-reporting being infected through medical procedures }\end{array}$ \\
\hline Skin penetration & $\begin{array}{l}\text { reporting intentional skin penetration (tattoo, scarring, or other practices) })^{\mathrm{a}} \text { OR having been exposed to another person's } \\
\text { blood through damaged skin or mucosa }{ }^{\mathrm{a}} \text { OR reporting being infected in an occupational or non-occupational accident } \\
\text { with skin penetration }\end{array}$ \\
\hline $\begin{array}{l}\text { Sexually transmitted } \\
\text { infections }\end{array}$ & $\begin{array}{l}\text { self-report on having HBV OR gonorrhea OR syphilis OR genital herpes OR proctitis OR other STI at any time before finding } \\
\text { out about HIV-positive status }\end{array}$ \\
\hline Exposure to HCV & positive test for anti-HCV antibodies \\
\hline Exposure to HBV & positive test for HBsAg OR positive test for AntiHBc antibody \\
\hline
\end{tabular}

aduring 10 years before finding out about HIV-positive status

prevalence in respective key populations [12] in the following hierarchy: injecting drug use, homosexual exposure between men, heterosexual exposure, and other. We did not create more detailed or mixed categories, such as those developed by CDC and other authors [17], to enable comparison with Ukrainian registered modes of transmission data. Instead, we present prevalence of all possible two risk factor combinations.

Participants who refused to answer one or more questions required for determining the survey-based mode of transmission were excluded from the analysis of modes of transmission but were retained in the dataset for analysis of other variables.

\section{Sensitivity analysis}

To assess the randomness of the sampling approach and thus the representativeness of the survey sample, we compared the registered modes of transmission distribution in the survey sample and the rest of the patients in the registry who did not participate in the survey. The significance of difference for each registered mode of transmission was tested using Chi-square tests.

\section{Hypotheses testing}

The main research question was whether there is a difference between the proportion of patients in corresponding registered modes of transmission and survey-based modes of transmission categories. Since these two variables were measured using the same participants and could be considered related, we used the McNemar test to determine the significance of difference between proportions for each major mode of transmission (heterosexual exposure, injecting drug use, homosexual exposure, and other). To assess the extent of agreement between the registration data and survey-based determination of mode of transmission on individual level, we computed Cohen's Kappa and corresponding $p$-value. We used the Mantel-Haenszel test for trend (for $2 \times \mathrm{r}$ tables) to test the significance of change in the proportion of main transmission categories and prevalence of risk factors over time [18].

To determine the accuracy of the official paper-based reporting system, we used the Chi-square test to determine difference in the distribution of the main modes of transmission between the reporting forms and the verified registry.

$P$-values less than 0.05 were considered statistically significant. Statistical analysis was done using SPSS for Windows version 23 (IBM Corporation, Armonk, NY USA).

\section{Extrapolation}

An adjusted distribution of modes of transmission among total national cases registered in 2015 was imputed by extrapolating the magnitude of misclassification observed in our sample. For each mode of transmission category, we calculated an extrapolation coefficient as a ratio of the proportion observed in the survey to the proportion in the registry. The coefficients were then applied to the reported number of cases within each category to calculate percentages representative of the adjusted modes of transmission among all HIV cases registered in 2015.

\section{Results}

\section{Recruitment}

A total of 3913 new HIV cases, excluding cases of motherto-child transmission, were reported in the seven study regions during the three October-December periods in 2013, 2014, and 2015 ( $N=1421,1209$, and 1283, respectively). 
We verified and extracted data from 3627 patient registration forms into the study registry. In two regions, Lviv and Dnipropetrovsk, data from deceased or transferred patients were unavailable for extraction. In three regions, the study team found more registration forms (up to 20\%) for patients registered within the study period than were included in the official registration reports.

Among the patients included in the registry, 9.2\% were deceased, $1.5 \%$ had moved outside of the study region, $2.1 \%$ were incarcerated, and $9.3 \%$ were lost to follow-up. Of 2567 patients who were contacted, 2285 agreed to participate in the study, yielding an $89.0 \%$ response rate. Overall, we recruited $63.0 \%(50.0-88.0 \%$ across the regions, data presented in Supplement Table S2) of all patients in the verified registry.

The sample consisted of $54.8 \%$ men and $45.2 \%$ women. Median age at registration was 35 years (standard deviation [SD], 8.85). Of the study participants, 2260 answered definitively all questions required to construct the survey-based modes of transmission variable.

\section{Sensitivity results}

Supplement Table S3 shows the distribution of registered modes of transmission in official reports and among patients in the registry who were and were not recruited. The recruited sample had a lower proportion of patients who were registered with homosexual exposure as the mode of transmission $(2.8 \%$ vs. $6.0 \%$ among those not recruited, $p<0.001)$. There were no significant differences in other categories.

\section{Degree of misclassification}

Table 2 shows the prevalence of risk factors within the four registered modes of transmission categories. More than one-third $(36.3 \%)$ of men registered with heterosexual exposure as the mode of transmission reported injecting drug use risk, $49.1 \%$ were $\mathrm{HCV}$ positive, and $7.8 \%$ reported having sex with men. Table 2 also shows the registered modes of transmission categories that were assigned to people with specific risk factors. For instance, only $37.7 \%$ of men reporting sex with men were registered with homosexual exposure as the mode of transmission. This percent ranged from $5.3 \%$ in the $\geq 45$-year age category to $66.7 \%$ in the $<25$ years category. Data disaggregated by region are presented in Supplement Table S4.

Most participants reported exposure to more than one risk factor. Prevalence of two-factor combinations is shown in Table 3. For instance, $76.9 \%$ of men who reported having sex with men also had heterosexual exposure, and $25.4 \%$ of them reported injecting drug use. Prevalence of anti-HCV antibodies was highest (82.3\%) among participants reporting injecting drug use, followed by those with HBV exposure (76.8\%), high-risk heterosexual exposure $(65.2 \%)$, skin penetration risk (56.7\%), and STI history (54.7\%).

Although registered modes of transmission and surveybased modes of transmission correlated (Table 4), the correlation was not perfect. Only $51.4 \%$ of patients who registered their mode of transmission as heterosexual were in the same category in our survey. Of the remaining patients with heterosexual exposure as the registered mode of transmission, $45.8 \%$ had injecting drug use exposure, and 2.9\% (6.9\% of men) were MSM who did not inject. The Kappa statistic, presented in Table 5 (with regional data in Supplement Table S5), indicates that the agreement between registered and survey-based results in the majority of categories is fair (0.3-0.4 on the scale from -1 to 1$)$. Slightly poorer agreement was observed among women, and higher agreement was for the homosexual mode of transmission.

The resulting distribution of registered modes of transmission and survey-based modes of transmission among survey participants showed significant differences in all four categories (Table 5). The proportion of cases attributable to injecting drug use was higher in our survey compared to registration records $(70.1 \%$ vs. $46.2 \%$ among men; $47.3 \%$ vs. $17.6 \%$ among women), the proportion of cases related to homosexual exposure was also higher (6.9\% vs. $4.7 \%)$, and the number of infections likely acquired through heterosexual transmission was lower (23.0\% vs. $48.2 \%$ among men; $52.1 \%$ vs. $80.7 \%$ among women). The degree of misclassification was proportionally greatest among injecting drug users (the true number of cases is underestimated by at least $44.5 \%$ ), followed by MSM (underestimated by at least 26.7\%). Together, this resulted in overestimation of the proportion of heterosexual exposure as the mode of transmission by at least $74.1 \%$. The degree of misclassification varied widely across regions, ranging from +17.8 to $+121.4 \%$ for heterosexual exposure, from $-21.4 \%$ to $-65.7 \%$ for injecting drug use, and from $+25.0 \%$ to $-100.0 \%$ for homosexual exposure (Supplement Table S5).

\section{Extrapolation results}

Adjusting for the magnitude of misclassification, we estimated that approximately $43.6 \%$ of all patients registered in 2015 in Ukraine had acquired HIV through heterosexual exposure (compared to $70.1 \%$ in the official Ministry of Health report), $52.1 \%$ had acquired HIV through injecting drug use (compared to $26.7 \%$ ), $4.2 \%$ had acquired HIV through homosexual exposure (compared to $2.9 \%$ ), and $0.1 \%$ through other modes of transmission (compared to $0.3 \%$ ).

\section{Trends over time}

We assessed the significance of trends in the registered modes of transmission categories, both in the official reports and in the verified registry, in survey-based modes 
Table 2 Prevalence of risk factors by registered mode of transmission, sex and age

\begin{tabular}{|c|c|c|c|c|c|c|c|c|c|c|c|}
\hline & \multirow{4}{*}{$\begin{array}{l}\text { Risk } \\
\text { factor }\end{array}$} & \multicolumn{10}{|c|}{ Registered mode of transmission } \\
\hline & & \multicolumn{2}{|l|}{$\overline{\mathrm{HET}}$} & \multicolumn{2}{|l|}{ IDU } & \multicolumn{2}{|l|}{ MSM } & \multicolumn{2}{|l|}{$\mathrm{OTH}$} & \multicolumn{2}{|l|}{ Total } \\
\hline & & $\mathrm{N}$ & Col \% & $\mathrm{N}$ & $\mathrm{Col} \%$ & $\bar{N}$ & $\mathrm{Col} \%$ & $\mathrm{~N}$ & $\mathrm{Col} \%$ & $\bar{N}$ & $\mathrm{Col} \%$ \\
\hline & & Row \% & & Row \% & & Row \% & & Row \% & & Row \% & \\
\hline \multirow[t]{20}{*}{ Men } & Total & 609 & $42.2 \%$ & 574 & $75.9 \%$ & 59 & $93.7 \%$ & 11 & $47.8 \%$ & 1253 & $54.8 \%$ \\
\hline & & $48.6 \%$ & & $45.8 \%$ & & $4.7 \%$ & & $0.9 \%$ & & $100.0 \%$ & \\
\hline & het & 590 & $96.9 \%$ & 563 & $98.1 \%$ & 39 & $66.1 \%$ & 11 & $100.0 \%$ & 1203 & $96.0 \%$ \\
\hline & & $49.0 \%$ & & $46.8 \%$ & & $3.2 \%$ & & $1.0 \%$ & & $100.0 \%$ & \\
\hline & hrh & 310 & $51.2 \%$ & 411 & $71.7 \%$ & 11 & $18.6 \%$ & 6 & $54.5 \%$ & 738 & $59.1 \%$ \\
\hline & & $42.0 \%$ & & $55.7 \%$ & & $1.5 \%$ & & $0.8 \%$ & & $100.0 \%$ & \\
\hline & sti & 221 & $38.2 \%$ & 239 & $44.4 \%$ & 31 & $53.4 \%$ & 3 & $27.3 \%$ & 494 & $41.7 \%$ \\
\hline & & $44.7 \%$ & & $48.4 \%$ & & $6.3 \%$ & & $0.6 \%$ & & $100.0 \%$ & \\
\hline & idu & 220 & $36.3 \%$ & 503 & $87.8 \%$ & 6 & $10.3 \%$ & 6 & $54.5 \%$ & 735 & $58.9 \%$ \\
\hline & & $29.9 \%$ & & $68.4 \%$ & & $0.8 \%$ & & $0.9 \%$ & & $100.0 \%$ & \\
\hline & hcv & 293 & $49.1 \%$ & 452 & $80.9 \%$ & 6 & $10.7 \%$ & 4 & $40.0 \%$ & 755 & $61.8 \%$ \\
\hline & & $38.8 \%$ & & $59.9 \%$ & & $0.8 \%$ & & $0.5 \%$ & & $100.0 \%$ & \\
\hline & hbv & 255 & $42.7 \%$ & 342 & $61.3 \%$ & 27 & $48.2 \%$ & 4 & $40.0 \%$ & 628 & $51.4 \%$ \\
\hline & & $40.6 \%$ & & $54.5 \%$ & & $4.3 \%$ & & $0.6 \%$ & & $100.0 \%$ & \\
\hline & $\mathrm{msm}$ & 46 & $7.8 \%$ & 34 & $6.1 \%$ & 49 & $83.1 \%$ & 1 & $9.1 \%$ & 130 & $10.6 \%$ \\
\hline & & $35.4 \%$ & & $26.2 \%$ & & $37.7 \%$ & & $0.7 \%$ & & $100.0 \%$ & \\
\hline & nos & 95 & $15.9 \%$ & 51 & $9.2 \%$ & 7 & $12.5 \%$ & 1 & $9.1 \%$ & 154 & $12.7 \%$ \\
\hline & & $61.7 \%$ & & $33.1 \%$ & & $4.5 \%$ & & $0.7 \%$ & & $100.0 \%$ & \\
\hline & pen & 360 & $64.6 \%$ & 398 & $74.8 \%$ & 21 & $43.8 \%$ & 10 & $90.9 \%$ & 789 & $68.7 \%$ \\
\hline & & $45.6 \%$ & & $50.4 \%$ & & $2.7 \%$ & & $1.3 \%$ & & $100.0 \%$ & \\
\hline \multirow[t]{20}{*}{ Women } & Total & 834 & $57.8 \%$ & 182 & $24.1 \%$ & 4 & $6.3 \%$ & 12 & $52.2 \%$ & 1032 & $45.2 \%$ \\
\hline & & $80.8 \%$ & & $17.6 \%$ & & $0.4 \%$ & & $1.2 \%$ & & $100.0 \%$ & \\
\hline & het & 824 & $98.8 \%$ & 177 & $97.3 \%$ & 4 & $100.0 \%$ & 11 & $91.7 \%$ & 1016 & $98.4 \%$ \\
\hline & & $81.1 \%$ & & $17.4 \%$ & & $0.4 \%$ & & $1.1 \%$ & & $100.0 \%$ & \\
\hline & hrh & 328 & $39.5 \%$ & 129 & $70.9 \%$ & 2 & $50.0 \%$ & 4 & $33.3 \%$ & 463 & $45.0 \%$ \\
\hline & & $70.8 \%$ & & $27.9 \%$ & & $0.4 \%$ & & $0.9 \%$ & & $100.0 \%$ & \\
\hline & sti & 286 & $36.3 \%$ & 83 & $49.7 \%$ & 1 & $50.0 \%$ & 4 & $36.4 \%$ & 374 & $38.6 \%$ \\
\hline & & $76.5 \%$ & & $22.2 \%$ & & $0.3 \%$ & & $1.0 \%$ & & $100.0 \%$ & \\
\hline & idu & 136 & $16.5 \%$ & 138 & $76.2 \%$ & 1 & $25.0 \%$ & 5 & $41.7 \%$ & 280 & $27.5 \%$ \\
\hline & & $48.6 \%$ & & $49.3 \%$ & & $0.4 \%$ & & $1.7 \%$ & & $100.0 \%$ & \\
\hline & hcv & 301 & $37.2 \%$ & 121 & $68.0 \%$ & 2 & $50.0 \%$ & 2 & $16.7 \%$ & 426 & $42.4 \%$ \\
\hline & & $70.7 \%$ & & $28.4 \%$ & & $0.5 \%$ & & $0.4 \%$ & & $100.0 \%$ & \\
\hline & hbv & 286 & $35.3 \%$ & 97 & $54.5 \%$ & 2 & $50.0 \%$ & 1 & $8.3 \%$ & 386 & $38.4 \%$ \\
\hline & & $74.1 \%$ & & $25.1 \%$ & & $0.5 \%$ & & $0.3 \%$ & & $100.0 \%$ & \\
\hline & msm & 0 & $0.0 \%$ & 0 & $0.0 \%$ & 0 & $0.0 \%$ & 0 & $0.0 \%$ & 0 & $0.0 \%$ \\
\hline & & $0.0 \%$ & & $0.0 \%$ & & $0.0 \%$ & & $0.0 \%$ & & $0.0 \%$ & \\
\hline & nos & 161 & $19.9 \%$ & 31 & $17.4 \%$ & 1 & $25.0 \%$ & 1 & $10.0 \%$ & 194 & $19.3 \%$ \\
\hline & & $83.0 \%$ & & $16.0 \%$ & & $0.5 \%$ & & $0.5 \%$ & & $100.0 \%$ & \\
\hline & pen & 423 & $54.3 \%$ & 119 & $72.1 \%$ & 3 & $75.0 \%$ & 4 & $40.0 \%$ & 549 & $57.3 \%$ \\
\hline & & $77.0 \%$ & & $21.7 \%$ & & $0.5 \%$ & & $0.8 \%$ & & $100.0 \%$ & \\
\hline
\end{tabular}


Table 2 Prevalence of risk factors by registered mode of transmission, sex and age (Continued)

\begin{tabular}{|c|c|c|c|c|c|c|c|c|c|c|c|}
\hline & \multirow{4}{*}{$\begin{array}{l}\text { Risk } \\
\text { factor }\end{array}$} & \multicolumn{10}{|c|}{ Registered mode of transmission } \\
\hline & & \multicolumn{2}{|l|}{ HET } & \multicolumn{2}{|l|}{ IDU } & \multicolumn{2}{|l|}{ MSM } & \multicolumn{2}{|l|}{$\mathrm{OTH}$} & \multicolumn{2}{|l|}{ Total } \\
\hline & & $\mathrm{N}$ & $\mathrm{Col} \%$ & $\bar{N}$ & $\mathrm{Col} \%$ & $\bar{N}$ & Col \% & $\bar{N}$ & $\mathrm{Col} \%$ & $\bar{N}$ & Col \% \\
\hline & & Row \% & & Row \% & & Row \% & & Row \% & & Row \% & \\
\hline \multirow[t]{20}{*}{$<=24$} & Total & 122 & $8.5 \%$ & 21 & $2.8 \%$ & 14 & $22.2 \%$ & 0 & $0.0 \%$ & 157 & $6.9 \%$ \\
\hline & & $77.7 \%$ & & $13.4 \%$ & & $8.9 \%$ & & $0.0 \%$ & & $100.0 \%$ & \\
\hline & het & 120 & $98.4 \%$ & 21 & $100.0 \%$ & 9 & $64.3 \%$ & 0 & $0.0 \%$ & 150 & $95.5 \%$ \\
\hline & & $80.0 \%$ & & $14.0 \%$ & & $6.0 \%$ & & $0.0 \%$ & & $100.0 \%$ & \\
\hline & hrh & 40 & $33.1 \%$ & 11 & $52.4 \%$ & 2 & $14.3 \%$ & 0 & $0.0 \%$ & 53 & $34.0 \%$ \\
\hline & & $75.5 \%$ & & $20.8 \%$ & & $3.7 \%$ & & $0.0 \%$ & & $100.0 \%$ & \\
\hline & sti & 34 & $30.6 \%$ & 5 & $29.4 \%$ & 7 & $53.8 \%$ & 0 & $0.0 \%$ & 46 & $32.6 \%$ \\
\hline & & $73.9 \%$ & & $10.9 \%$ & & $15.2 \%$ & & $0.0 \%$ & & $100.0 \%$ & \\
\hline & idu & 9 & $7.5 \%$ & 11 & $55.0 \%$ & 0 & $0.0 \%$ & 0 & $0.0 \%$ & 20 & $13.1 \%$ \\
\hline & & $45.0 \%$ & & $55.0 \%$ & & $0.0 \%$ & & $0.0 \%$ & & $100.0 \%$ & \\
\hline & hcv & 31 & $25.6 \%$ & 10 & $47.6 \%$ & 0 & $0.0 \%$ & 0 & $0.0 \%$ & 41 & $26.3 \%$ \\
\hline & & $75.6 \%$ & & $24.4 \%$ & & $0.0 \%$ & & $0.0 \%$ & & $100.0 \%$ & \\
\hline & hbv & 31 & $25.6 \%$ & 9 & $42.9 \%$ & 7 & $50.0 \%$ & 0 & $0.0 \%$ & 47 & $30.1 \%$ \\
\hline & & $66.0 \%$ & & $19.1 \%$ & & $14.9 \%$ & & $0.0 \%$ & & $100.0 \%$ & \\
\hline & $\mathrm{msm}$ & 6 & $24.0 \%$ & 0 & $0.0 \%$ & 12 & $85.7 \%$ & 0 & $0.0 \%$ & 18 & $39.1 \%$ \\
\hline & & $33.3 \%$ & & $0.0 \%$ & & $66.7 \%$ & & $0.0 \%$ & & $100.0 \%$ & \\
\hline & nos & 19 & $16.5 \%$ & 1 & $4.8 \%$ & 2 & $14.3 \%$ & 0 & $0.0 \%$ & 22 & $14.7 \%$ \\
\hline & & $86.4 \%$ & & $4.5 \%$ & & $9.1 \%$ & & $0.0 \%$ & & $100.0 \%$ & \\
\hline & pen & 58 & $50.9 \%$ & 14 & $66.7 \%$ & 4 & $33.3 \%$ & 0 & $0.0 \%$ & 76 & $51.7 \%$ \\
\hline & & $76.3 \%$ & & $18.4 \%$ & & $5.3 \%$ & & $0.0 \%$ & & $100.0 \%$ & \\
\hline \multirow[t]{20}{*}{$25-44$} & Total & 1023 & $70.9 \%$ & 626 & $82.8 \%$ & 45 & $71.4 \%$ & 18 & $78.3 \%$ & 1712 & $74.9 \%$ \\
\hline & & $59.8 \%$ & & $36.6 \%$ & & $2.6 \%$ & & $1.0 \%$ & & $100.0 \%$ & \\
\hline & het & 1013 & $99.0 \%$ & 618 & $98.7 \%$ & 31 & $68.9 \%$ & 18 & $100.0 \%$ & 1680 & $98.1 \%$ \\
\hline & & $60.3 \%$ & & $36.8 \%$ & & $1.8 \%$ & & $1.1 \%$ & & $100.0 \%$ & \\
\hline & hrh & 482 & $47.2 \%$ & 457 & $73.1 \%$ & 10 & $22.2 \%$ & 9 & $50.0 \%$ & 958 & $56.1 \%$ \\
\hline & & $50.3 \%$ & & $47.7 \%$ & & $1.0 \%$ & & $1.0 \%$ & & $100.0 \%$ & \\
\hline & sti & 368 & $37.8 \%$ & 252 & $43.2 \%$ & 22 & $50.0 \%$ & 4 & $23.5 \%$ & 646 & $39.9 \%$ \\
\hline & & $57.0 \%$ & & $39.0 \%$ & & $3.4 \%$ & & $0.6 \%$ & & $100.0 \%$ & \\
\hline & idu & 265 & $26.1 \%$ & 545 & $87.1 \%$ & 6 & $13.3 \%$ & 8 & $44.4 \%$ & 824 & $48.4 \%$ \\
\hline & & $32.2 \%$ & & $66.1 \%$ & & $0.7 \%$ & & $1.0 \%$ & & $100.0 \%$ & \\
\hline & hcv & 435 & $43.6 \%$ & 489 & $80.3 \%$ & 7 & $16.7 \%$ & 5 & $29.4 \%$ & 936 & $56.2 \%$ \\
\hline & & $46.5 \%$ & & $52.2 \%$ & & $0.7 \%$ & & $0.6 \%$ & & $100.0 \%$ & \\
\hline & hbv & 383 & $38.4 \%$ & 365 & $60.0 \%$ & 19 & $45.2 \%$ & 2 & $11.8 \%$ & 769 & $46.2 \%$ \\
\hline & & $49.8 \%$ & & $47.5 \%$ & & $2.5 \%$ & & $0.2 \%$ & & $100.0 \%$ & \\
\hline & $\mathrm{msm}$ & 26 & $6.2 \%$ & 30 & $6.2 \%$ & 36 & $85.7 \%$ & 1 & $12.5 \%$ & 93 & $9.8 \%$ \\
\hline & & $28.0 \%$ & & $32.3 \%$ & & $38.7 \%$ & & $1.0 \%$ & & $100.0 \%$ & \\
\hline & nos & 163 & $16.3 \%$ & 65 & $10.7 \%$ & 6 & $14.0 \%$ & 1 & $6.3 \%$ & 235 & $14.1 \%$ \\
\hline & & $69.4 \%$ & & $27.7 \%$ & & $2.6 \%$ & & $0.3 \%$ & & $100.0 \%$ & \\
\hline & pen & 569 & $60.1 \%$ & 432 & $74.7 \%$ & 18 & $50.0 \%$ & 11 & $68.8 \%$ & 1030 & $65.4 \%$ \\
\hline & & $55.2 \%$ & & $41.9 \%$ & & $1.7 \%$ & & $1.2 \%$ & & $100.0 \%$ & \\
\hline
\end{tabular}


Table 2 Prevalence of risk factors by registered mode of transmission, sex and age (Continued)

\begin{tabular}{|c|c|c|c|c|c|c|c|c|c|c|c|}
\hline & \multirow{4}{*}{$\begin{array}{l}\text { Risk } \\
\text { factor }\end{array}$} & \multicolumn{10}{|c|}{ Registered mode of transmission } \\
\hline & & \multicolumn{2}{|l|}{ HET } & \multicolumn{2}{|l|}{ IDU } & \multicolumn{2}{|l|}{ MSM } & \multicolumn{2}{|l|}{$\mathrm{OTH}$} & \multicolumn{2}{|l|}{ Total } \\
\hline & & $\mathrm{N}$ & $\mathrm{Col} \%$ & $\mathrm{~N}$ & $\mathrm{Col} \%$ & $\mathrm{~N}$ & $\mathrm{Col} \%$ & N & $\mathrm{Col} \%$ & $\mathrm{~N}$ & $\mathrm{Col} \%$ \\
\hline & & Row \% & & Row \% & & Row \% & & Row \% & & Row \% & \\
\hline \multirow[t]{20}{*}{$>=45$} & Total & 298 & $20.7 \%$ & 109 & $14.4 \%$ & 4 & $6.3 \%$ & 5 & $21.7 \%$ & 416 & $18.2 \%$ \\
\hline & & $71.6 \%$ & & $26.2 \%$ & & $1.0 \%$ & & $1.2 \%$ & & $100.0 \%$ & \\
\hline & het & 281 & $94.3 \%$ & 101 & $92.7 \%$ & 3 & $75.0 \%$ & 4 & $80.0 \%$ & 389 & $93.5 \%$ \\
\hline & & $72.2 \%$ & & $26.0 \%$ & & $0.8 \%$ & & $1.0 \%$ & & $100.0 \%$ & \\
\hline & hrh & 116 & $39.5 \%$ & 72 & $66.1 \%$ & 1 & $25.0 \%$ & 1 & $20.0 \%$ & 190 & $46.1 \%$ \\
\hline & & $61.1 \%$ & & $37.9 \%$ & & $0.5 \%$ & & $0.5 \%$ & & $100.0 \%$ & \\
\hline & sti & 105 & $37.4 \%$ & 65 & $62.5 \%$ & 3 & $100.0 \%$ & 3 & $60.0 \%$ & 176 & $44.8 \%$ \\
\hline & & $59.7 \%$ & & $36.9 \%$ & & $1.7 \%$ & & $1.7 \%$ & & $100.0 \%$ & \\
\hline & idu & 82 & $27.8 \%$ & 85 & $78.7 \%$ & 1 & $25.0 \%$ & 3 & $60.0 \%$ & 171 & $41.5 \%$ \\
\hline & & $48.0 \%$ & & $49.7 \%$ & & $0.6 \%$ & & $1.7 \%$ & & $100.0 \%$ & \\
\hline & hcv & 128 & $44.4 \%$ & 74 & $69.2 \%$ & 1 & $25.0 \%$ & 1 & $20.0 \%$ & 204 & $50.5 \%$ \\
\hline & & $62.7 \%$ & & $36.3 \%$ & & $0.5 \%$ & & $0.5 \%$ & & $100.0 \%$ & \\
\hline & hbv & 127 & $44.1 \%$ & 65 & $60.7 \%$ & 3 & $75.0 \%$ & 3 & $60.0 \%$ & 198 & $49.0 \%$ \\
\hline & & $64.1 \%$ & & $32.8 \%$ & & $1.5 \%$ & & $1.6 \%$ & & $100.0 \%$ & \\
\hline & msm & 14 & $9.4 \%$ & 4 & $5.6 \%$ & 1 & $33.3 \%$ & 0 & $0.0 \%$ & 19 & $8.4 \%$ \\
\hline & & $73.7 \%$ & & $21.1 \%$ & & $5.2 \%$ & & $0.0 \%$ & & $100.0 \%$ & \\
\hline & nos & 74 & $25.1 \%$ & 16 & $15.4 \%$ & 0 & $0.0 \%$ & 1 & $20.0 \%$ & 91 & $22.4 \%$ \\
\hline & & $81.3 \%$ & & $17.6 \%$ & & $0.0 \%$ & & $1.1 \%$ & & $100.0 \%$ & \\
\hline & pen & 156 & $56.5 \%$ & 71 & $72.4 \%$ & 2 & $50.0 \%$ & 3 & $60.0 \%$ & 232 & $60.6 \%$ \\
\hline & & $67.2 \%$ & & $30.6 \%$ & & $0.9 \%$ & & $1.3 \%$ & & $100.0 \%$ & \\
\hline \multirow[t]{20}{*}{ Total } & & 1443 & $100.0 \%$ & 756 & $100.0 \%$ & 63 & $100.0 \%$ & 23 & $100.0 \%$ & 2285 & $100.0 \%$ \\
\hline & & $63.2 \%$ & & $33.1 \%$ & & $2.8 \%$ & & $0.9 \%$ & & $100.0 \%$ & \\
\hline & het & 1414 & $98.0 \%$ & 740 & $97.9 \%$ & 43 & $68.3 \%$ & 22 & $95.7 \%$ & 2219 & $97.1 \%$ \\
\hline & & $63.7 \%$ & & $33.3 \%$ & & $1.9 \%$ & & $1.1 \%$ & & $100.0 \%$ & \\
\hline & hrh & 638 & $44.4 \%$ & 540 & $71.5 \%$ & 13 & $20.6 \%$ & 10 & $43.5 \%$ & 1201 & $52.7 \%$ \\
\hline & & $53.1 \%$ & & $45.0 \%$ & & $1.1 \%$ & & $0.8 \%$ & & $100.0 \%$ & \\
\hline & sti & 507 & $37.1 \%$ & 322 & $45.7 \%$ & 32 & $53.3 \%$ & 7 & $31.8 \%$ & 868 & $40.3 \%$ \\
\hline & & $58.4 \%$ & & $37.1 \%$ & & $3.7 \%$ & & $0.8 \%$ & & $100.0 \%$ & \\
\hline & idu & 356 & $24.9 \%$ & 641 & $85.0 \%$ & 7 & $11.3 \%$ & 11 & $47.8 \%$ & 1015 & $44.8 \%$ \\
\hline & & $35.1 \%$ & & $63.2 \%$ & & $0.7 \%$ & & $1.0 \%$ & & $100.0 \%$ & \\
\hline & hcv & 594 & $42.2 \%$ & 573 & $77.7 \%$ & 8 & $13.3 \%$ & 6 & $27.3 \%$ & 1181 & $53.1 \%$ \\
\hline & & $50.3 \%$ & & $48.5 \%$ & & $0.7 \%$ & & $0.5 \%$ & & $100.0 \%$ & \\
\hline & hbv & 541 & $38.5 \%$ & 439 & $59.6 \%$ & 29 & $48.3 \%$ & 5 & $22.7 \%$ & 1014 & $45.6 \%$ \\
\hline & & $53.4 \%$ & & $43.3 \%$ & & $2.9 \%$ & & $0.4 \%$ & & $100.0 \%$ & \\
\hline & msm & 46 & $7.8 \%$ & 34 & $6.1 \%$ & 49 & $83.1 \%$ & 1 & $9.1 \%$ & 130 & $10.6 \%$ \\
\hline & & $35.4 \%$ & & $26.2 \%$ & & $37.7 \%$ & & $0.7 \%$ & & $100.0 \%$ & \\
\hline & nos & 256 & $18.2 \%$ & 82 & $11.2 \%$ & 8 & $13.3 \%$ & 2 & $9.5 \%$ & 348 & $15.7 \%$ \\
\hline & & $73.6 \%$ & & $23.6 \%$ & & $2.3 \%$ & & $0.5 \%$ & & $100.0 \%$ & \\
\hline & pen & 783 & $58.6 \%$ & 517 & $74.2 \%$ & 24 & $46.2 \%$ & 14 & $66.7 \%$ & 1338 & $63.5 \%$ \\
\hline & & $58.5 \%$ & & $38.6 \%$ & & $1.8 \%$ & & $1.1 \%$ & & $100.0 \%$ & \\
\hline
\end{tabular}

Risk factors (for definitions see Table 1): het heterosexual exposure, hrh high-risk heterosexual exposure, sti sexually transmitted infections, idu injecting drug use, $h c v$ exposure to $\mathrm{HCV}$, $h b v$ exposure to $\mathrm{HBV}$, msm homosexual exposure, nos nosocomial exposure, pen skin penetration exposure Modes of transmission: HET heterosexual, IDU injecting drug use, MSM homosexual, OTH other 


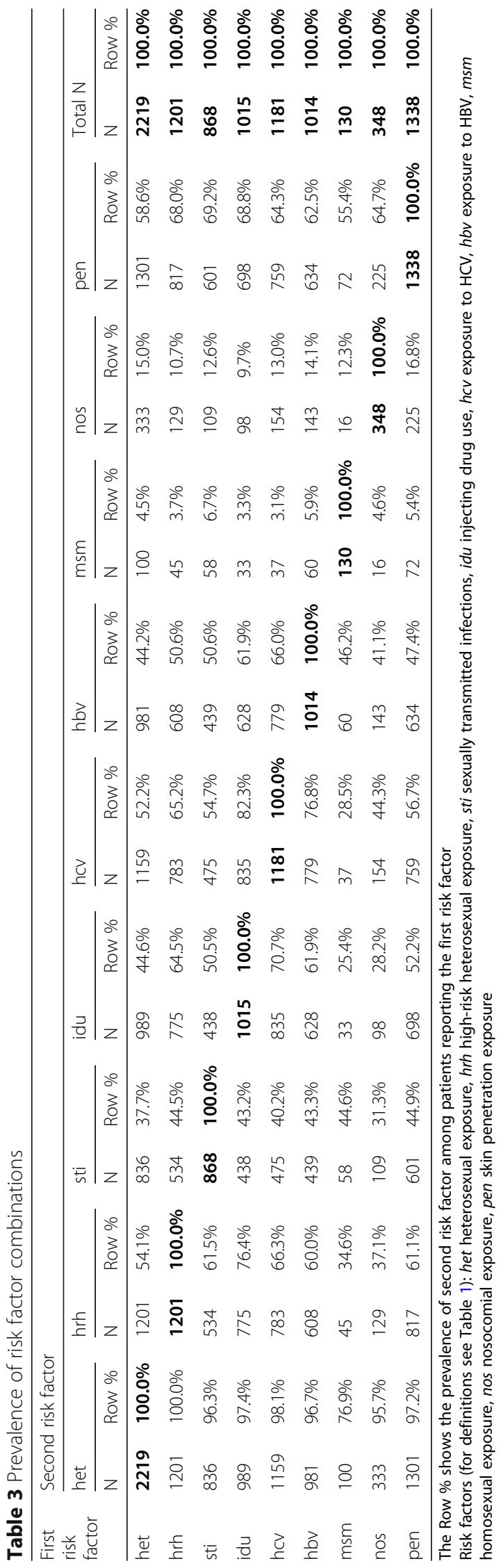


Table 4 Cross-tabulation of registered and survey-based mode of transmission by age and sex

\begin{tabular}{|c|c|c|c|c|c|c|c|c|c|c|c|}
\hline & \multirow{4}{*}{$\begin{array}{l}\text { Survey- } \\
\text { based } \\
\text { mode of } \\
\text { transmission }\end{array}$} & \multicolumn{10}{|c|}{ Registered mode of transmission } \\
\hline & & \multicolumn{2}{|l|}{ HET } & \multicolumn{2}{|l|}{ IDU } & \multicolumn{2}{|l|}{ MSM } & \multicolumn{2}{|l|}{ OTH } & \multicolumn{2}{|l|}{ Total } \\
\hline & & N & $\mathrm{Col} \%$ & N & $\mathrm{Col} \%$ & $\mathrm{~N}$ & $\mathrm{Col} \%$ & $\mathrm{~N}$ & $\mathrm{Col} \%$ & $\mathrm{~N}$ & $\mathrm{Col} \%$ \\
\hline & & Row \% & & Row \% & & Row \% & & Row \% & & Row \% & \\
\hline \multirow[t]{8}{*}{ Men } & HET & 240 & $39.9 \%$ & 39 & $6.8 \%$ & 5 & $8.5 \%$ & 3 & $27.3 \%$ & 287 & $23.0 \%$ \\
\hline & & $83.6 \%$ & & $13.6 \%$ & & $1.7 \%$ & & $1.1 \%$ & & $100.0 \%$ & \\
\hline & IDU & 327 & $54.3 \%$ & 530 & $92.3 \%$ & 9 & $15.3 \%$ & 7 & $63.6 \%$ & 873 & $70.1 \%$ \\
\hline & & $37.5 \%$ & & $60.7 \%$ & & $1.0 \%$ & & $0.8 \%$ & & $100.0 \%$ & \\
\hline & MSM & 35 & $5.8 \%$ & 5 & $0.9 \%$ & 45 & $76.3 \%$ & 1 & $9.1 \%$ & 86 & $6.9 \%$ \\
\hline & & $40.7 \%$ & & $5.8 \%$ & & $52.3 \%$ & & $1.2 \%$ & & $100.0 \%$ & \\
\hline & UNK & 0 & $0.0 \%$ & 0 & $0.0 \%$ & 0 & $0.0 \%$ & 0 & $0.0 \%$ & 0 & $0.0 \%$ \\
\hline & & $0.0 \%$ & & $0.0 \%$ & & $0.0 \%$ & & $100.0 \%$ & & $0.0 \%$ & \\
\hline \multirow[t]{6}{*}{ Women } & HET & 498 & $59.7 \%$ & 33 & $18.1 \%$ & 1 & $25.0 \%$ & 6 & $50.0 \%$ & 538 & $52.1 \%$ \\
\hline & & $92.6 \%$ & & $6.1 \%$ & & $0.2 \%$ & & $1.1 \%$ & & $100.0 \%$ & \\
\hline & IDU & 331 & $39.7 \%$ & 149 & $81.9 \%$ & 3 & $75.0 \%$ & 5 & $41.7 \%$ & 488 & $47.3 \%$ \\
\hline & & $67.8 \%$ & & $30.5 \%$ & & $0.6 \%$ & & $1.1 \%$ & & $100.0 \%$ & \\
\hline & UNK & 5 & $0.6 \%$ & 0 & $0.0 \%$ & 0 & $0.0 \%$ & 1 & $8.3 \%$ & 6 & $0.6 \%$ \\
\hline & & $83.3 \%$ & & $0.0 \%$ & & $0.0 \%$ & & $16.7 \%$ & & $100.0 \%$ & \\
\hline \multirow[t]{8}{*}{$<=24$} & HET & 83 & $68.6 \%$ & 6 & $28.6 \%$ & 2 & $14.3 \%$ & 0 & $0.0 \%$ & 91 & $58.3 \%$ \\
\hline & & $91.2 \%$ & & $6.6 \%$ & & $2.2 \%$ & & $0.0 \%$ & & $100.0 \%$ & \\
\hline & IDU & 32 & $26.4 \%$ & 15 & $71.4 \%$ & 0 & $0.0 \%$ & 0 & $0.0 \%$ & 47 & $30.1 \%$ \\
\hline & & $68.1 \%$ & & $31.9 \%$ & & $0.0 \%$ & & $0.0 \%$ & & $100.0 \%$ & \\
\hline & MSM & 6 & $5.0 \%$ & 0 & $0.0 \%$ & 12 & $85.7 \%$ & 0 & $0.0 \%$ & 18 & $11.5 \%$ \\
\hline & & $33.3 \%$ & & $0.0 \%$ & & $66.7 \%$ & & $0.0 \%$ & & $100.0 \%$ & \\
\hline & UNK & 0 & $0.0 \%$ & 0 & $0.0 \%$ & 0 & $0.0 \%$ & 0 & $0.0 \%$ & 0 & $0.0 \%$ \\
\hline & & $0.0 \%$ & & $0.0 \%$ & & $0.0 \%$ & & $100.0 \%$ & & $0.0 \%$ & \\
\hline \multirow[t]{8}{*}{$25-44$} & HET & 519 & $50.8 \%$ & 52 & $8.3 \%$ & 2 & $4.4 \%$ & 8 & $44.4 \%$ & 581 & $34.0 \%$ \\
\hline & & $89.3 \%$ & & $9.0 \%$ & & $0.3 \%$ & & $1.4 \%$ & & $100.0 \%$ & \\
\hline & IDU & 482 & $47.2 \%$ & 571 & $91.2 \%$ & 11 & $24.4 \%$ & 9 & $50.0 \%$ & 1073 & $62.7 \%$ \\
\hline & & $44.9 \%$ & & $53.2 \%$ & & $1.0 \%$ & & $0.9 \%$ & & $100.0 \%$ & \\
\hline & MSM & 19 & $1.9 \%$ & 3 & $0.5 \%$ & 32 & $71.1 \%$ & 1 & $5.6 \%$ & 55 & $3.2 \%$ \\
\hline & & $34.5 \%$ & & $5.5 \%$ & & $58.2 \%$ & & $1.8 \%$ & & $100.0 \%$ & \\
\hline & UNK & 1 & $0.1 \%$ & 0 & $0.0 \%$ & 0 & $0.0 \%$ & 0 & $0.0 \%$ & 1 & $0.1 \%$ \\
\hline & & $100.0 \%$ & & $0.0 \%$ & & $0.0 \%$ & & $0.0 \%$ & & $100.0 \%$ & \\
\hline \multirow[t]{8}{*}{$>=45$} & HET & 136 & $46.3 \%$ & 14 & $12.8 \%$ & 2 & $50.0 \%$ & 1 & $20.0 \%$ & 153 & $37.1 \%$ \\
\hline & & $88.9 \%$ & & $9.2 \%$ & & $1.3 \%$ & & $0.6 \%$ & & $100.0 \%$ & \\
\hline & IDU & 144 & $49.0 \%$ & 93 & $85.3 \%$ & 1 & $25.0 \%$ & 3 & $60.0 \%$ & 241 & $58.5 \%$ \\
\hline & & $59.8 \%$ & & $38.6 \%$ & & $0.4 \%$ & & $1.2 \%$ & & $100.0 \%$ & \\
\hline & MSM & 10 & $3.4 \%$ & 2 & $1.8 \%$ & 1 & $25.0 \%$ & 0 & $0.0 \%$ & 13 & $3.2 \%$ \\
\hline & & $76.9 \%$ & & $15.4 \%$ & & $7.7 \%$ & & $0.0 \%$ & & $100.0 \%$ & \\
\hline & UNK & 4 & $1.4 \%$ & 0 & $0.0 \%$ & 0 & $0.0 \%$ & 1 & $20.0 \%$ & 5 & $1.2 \%$ \\
\hline & & $80.0 \%$ & & $0.0 \%$ & & $0.0 \%$ & & $20.0 \%$ & & $100.0 \%$ & \\
\hline \multirow[t]{3}{*}{ Total } & HET & 738 & $51.4 \%$ & 72 & $9.5 \%$ & 6 & $9.5 \%$ & 9 & $39.1 \%$ & 825 & $36.2 \%$ \\
\hline & & $89.5 \%$ & & $8.7 \%$ & & $0.7 \%$ & & $1.1 \%$ & & $100.0 \%$ & \\
\hline & IDU & 658 & $45.8 \%$ & 679 & $89.8 \%$ & 12 & $19.0 \%$ & 12 & $52.2 \%$ & 1361 & $59.7 \%$ \\
\hline
\end{tabular}


Table 4 Cross-tabulation of registered and survey-based mode of transmission by age and sex (Continued)

\begin{tabular}{|c|c|c|c|c|c|c|c|c|c|c|}
\hline \multirow{4}{*}{$\begin{array}{l}\text { Survey- } \\
\text { based } \\
\text { mode of } \\
\text { transmission }\end{array}$} & \multicolumn{10}{|c|}{ Registered mode of transmission } \\
\hline & \multicolumn{2}{|l|}{$\overline{\mathrm{HET}}$} & \multicolumn{2}{|l|}{ IDU } & \multicolumn{2}{|l|}{ MSM } & \multicolumn{2}{|l|}{$\mathrm{OTH}$} & \multicolumn{2}{|l|}{ Total } \\
\hline & $\bar{N}$ & $\mathrm{Col} \%$ & $\bar{N}$ & Col \% & $\bar{N}$ & $\mathrm{Col} \%$ & $\bar{N}$ & $\mathrm{Col} \%$ & $\mathrm{~N}$ & $\mathrm{Col} \%$ \\
\hline & \multicolumn{2}{|l|}{ Row \% } & \multicolumn{2}{|l|}{ Row \% } & \multicolumn{2}{|l|}{ Row \% } & \multicolumn{2}{|l|}{ Row \% } & \multicolumn{2}{|l|}{ Row \% } \\
\hline & $48.3 \%$ & & $49.9 \%$ & & $0.9 \%$ & & $0.9 \%$ & & $100.0 \%$ & \\
\hline \multirow[t]{2}{*}{ MSM } & 35 & $2.4 \%$ & 5 & $0.7 \%$ & 45 & $71.4 \%$ & 1 & $4.3 \%$ & 86 & $3.8 \%$ \\
\hline & $40.7 \%$ & & $5.8 \%$ & & $52.3 \%$ & & $1.2 \%$ & & $100.0 \%$ & \\
\hline \multirow[t]{2}{*}{ UNK } & 5 & $0.3 \%$ & 0 & $0.0 \%$ & 0 & $0.0 \%$ & 1 & $4.3 \%$ & 6 & $0.3 \%$ \\
\hline & $83.3 \%$ & & $0.0 \%$ & & $0.0 \%$ & & $16.7 \%$ & & $100.0 \%$ & \\
\hline
\end{tabular}

Modes of transmission: HET heterosexual, IDU injecting drug use, MSM homosexual, OTH other

Table 5 Misclassification of modes of transmission by sex and age

\begin{tabular}{|c|c|c|c|c|c|c|c|c|c|c|}
\hline & & \multicolumn{2}{|c|}{ Registry (verified records) } & \multicolumn{2}{|c|}{ Survey } & \multirow{2}{*}{$\begin{array}{l}\text { McNemar } \\
p \text {-value }\end{array}$} & \multirow[t]{2}{*}{ Kappa } & \multirow{2}{*}{$\begin{array}{l}\text { Kappa } \\
p \text {-value }\end{array}$} & \multirow[t]{2}{*}{ Misclassification } & \multirow{2}{*}{$\begin{array}{l}\text { Extrapolation } \\
\text { coefficient }\end{array}$} \\
\hline & & $\overline{\mathrm{N}}$ & MoT \% & $\bar{N}$ & MoT \% & & & & & \\
\hline \multirow[t]{5}{*}{ Men } & & & & & & & 0.33 & $<0.001$ & & \\
\hline & HET & 602 & $48.3 \%$ & 287 & $23.0 \%$ & $<0.001$ & 0.33 & $<0.001$ & $109.8 \%$ & $47.7 \%$ \\
\hline & IDU & 574 & $46.1 \%$ & 873 & $70.1 \%$ & $<0.001$ & 0.40 & $<0.001$ & $-34.2 \%$ & $152.1 \%$ \\
\hline & MSM & 59 & $4.7 \%$ & 86 & $6.9 \%$ & $<0.001$ & 0.60 & $<0.001$ & $-31.4 \%$ & $145.8 \%$ \\
\hline & UNK & 11 & $0.9 \%$ & 0 & $0.0 \%$ & 0.001 & 0.00 & $<0.001$ & $0.0 \%$ & $0.0 \%$ \\
\hline \multirow[t]{5}{*}{ Women } & & & & & & & 0.25 & $<0.001$ & & \\
\hline & HET & 834 & $80.8 \%$ & 538 & $52.1 \%$ & $<0.001$ & 0.25 & $<0.001$ & $55.0 \%$ & $64.5 \%$ \\
\hline & IDU & 182 & $17.6 \%$ & 488 & $47.3 \%$ & $<0.001$ & 0.25 & $<0.001$ & $-62.7 \%$ & $268.1 \%$ \\
\hline & $\mathrm{MSM}^{\mathrm{a}}$ & 4 & $0.4 \%$ & 0 & $0.0 \%$ & 0.125 & 0.00 & $<0.001$ & $0.0 \%$ & $0.0 \%$ \\
\hline & UNK & 12 & $1.2 \%$ & 6 & $0.6 \%$ & 0.210 & 0.10 & $<0.001$ & $100.0 \%$ & $50.0 \%$ \\
\hline \multirow[t]{5}{*}{$<=24$} & & & & & & & 0.35 & $<0.001$ & & \\
\hline & HET & 121 & $77.6 \%$ & 91 & $58.3 \%$ & $<0.001$ & 0.35 & $<0.001$ & $33.0 \%$ & $75.2 \%$ \\
\hline & IDU & 21 & $13.5 \%$ & 47 & $30.1 \%$ & $<0.001$ & 0.31 & $<0.001$ & $-55.3 \%$ & $223.8 \%$ \\
\hline & MSM & 14 & $9.0 \%$ & 18 & $11.5 \%$ & 0.289 & 0.72 & $<0.001$ & $-22.2 \%$ & $128.6 \%$ \\
\hline & UNK & 0 & $0.0 \%$ & 0 & $0.0 \%$ & $<0.001$ & 0.00 & $<0.001$ & $0.0 \%$ & $0.0 \%$ \\
\hline \multirow[t]{5}{*}{$25-44$} & & & & & & & 0.38 & $<0.001$ & & \\
\hline & HET & 1021 & $59.7 \%$ & 581 & $34.0 \%$ & $<0.001$ & 0.38 & $<0.001$ & $75.7 \%$ & $56.9 \%$ \\
\hline & IDU & 626 & $36.6 \%$ & 1073 & $62.7 \%$ & $<0.001$ & 0.39 & $<0.001$ & $-41.7 \%$ & $171.4 \%$ \\
\hline & MSM & 45 & $2.6 \%$ & 55 & $3.2 \%$ & 0.134 & 0.63 & $<0.001$ & $-18.2 \%$ & $122.2 \%$ \\
\hline & UNK & 18 & $1.1 \%$ & 1 & $0.1 \%$ & $<0.001$ & 0.00 & 0.918 & $1700.0 \%$ & $5.6 \%$ \\
\hline \multirow[t]{5}{*}{$>=45$} & & & & & & & 0.23 & $<0.001$ & & \\
\hline & HET & 294 & $71.4 \%$ & 153 & $37.1 \%$ & $<0.001$ & 0.23 & $<0.001$ & $92.2 \%$ & $52.0 \%$ \\
\hline & IDU & 109 & $26.5 \%$ & 241 & $58.5 \%$ & $<0.001$ & 0.26 & $<0.001$ & $-54.8 \%$ & $221.1 \%$ \\
\hline & MSM & 4 & $1.0 \%$ & 13 & $3.2 \%$ & 0.035 & 0.10 & 0.012 & $-69.2 \%$ & $325.0 \%$ \\
\hline & UNK & 5 & $1.2 \%$ & 5 & $1.2 \%$ & 1.000 & 0.19 & $<0.001$ & $0.0 \%$ & $100.0 \%$ \\
\hline \multirow[t]{5}{*}{ Grand Total } & & & & & & & 0.37 & $<0.001$ & & \\
\hline & HET & 1436 & $63.0 \%$ & 825 & $36.2 \%$ & $<0.001$ & 0.36 & $<0.001$ & $74.1 \%$ & $57.5 \%$ \\
\hline & IDU & 756 & $33.2 \%$ & 1361 & $59.7 \%$ & $<0.001$ & 0.37 & $<0.001$ & $-44.5 \%$ & $180.0 \%$ \\
\hline & MSM & 63 & $2.8 \%$ & 86 & $3.8 \%$ & 0.004 & 0.59 & $<0.001$ & $-26.7 \%$ & $136.5 \%$ \\
\hline & UNK & 23 & $1.0 \%$ & 6 & $0.3 \%$ & 0.002 & 0.07 & $<0.001$ & $283.3 \%$ & $26.1 \%$ \\
\hline
\end{tabular}


of transmission categories, and in individual risk factors (Tables 6, 7, 8 and 9). The proportion of the three main registered modes of transmission categories (heterosexual exposure, injecting drug use, and homosexual exposure) did not significantly change over time in either the official reports or the registry. The proportion of cases in the "other" category significantly decreased in the official reports (1.1 to $0.5 \%$ to $0.4 \%$ in 2013, 2014, and 2015 respectively; $p=0.019$ ), but this trend was not confirmed in the registry. The proportion of cases likely attributed to homosexual exposure increased significantly according to the survey-based modes of transmission results, from $2.5 \%$ (2013) to $3.5 \%$ (2014) to $5.2 \%$ (2015; $p=0.005)$. This increase was particularly striking in the $<25$-year age group with more than 6-time increase over the study period to $23.2 \%$ of all men and women in this group in 2015. The increase in cases attributed to homosexual exposure in two cities, Kyiv and Lviv, largely drove the overall trend (Supplement Tables S6, S7, S8 and S9). The proportion of cases attributed to injecting drug use as a survey-based mode of transmission decreased significantly, from $63.2 \%$ (2013) to $58.6 \%$ (2014) to $57.5 \%$ (2015; $p=$ 0.022 ). The proportion of cases attributed to heterosexual transmission was stable at slightly above $37 \%$ in 2014 and 2015 . Figure 1 displays the trends disaggregated by age and sex.

Several individual risk factors changed significantly from 2013 to 2015. The prevalence of heterosexual exposure decreased modestly but significantly, from $98.7 \%$ (2013) to $97.2 \%(2014)$ to $95.6 \%(2015 ; p<0.001)$, whereas high-risk heterosexual exposure decreased steeply, from $55.5 \%$ (2013) to $52.6 \%$ (2014) to $50.3 \%$ (2015), but this trend did not reach significance ( $p=$ 0.057). Self-reported injecting drug use risk decreased insignificantly among men from $60.0 \%$ (2013) to $60.9 \%$ (2014) to $56.3 \%$ (2015) and among women from $31.1 \%$ (2013) to $25.2 \%$ (2014) to $25.8 \%$ (2015). HCV seropositivity fluctuated among men from $61.4 \%$ (2013) to $65.4 \%$ (2014) to $59.3 \%$ (2015) and decreased significantly among women from $47.4 \%$ (2013) to $41.8 \%$ (2014) to $38.1 \%(2015 ; p=0.023)$. The proportion of men reporting sex with other men more than doubled, from $6.4 \%$ (2013) to $10.5 \%(2014)$ to $14.5 \%(2015 ; p<0.001)$. This

Table 6 Trends in modes of transmission by sex and age in the official reports

\begin{tabular}{lllllllll}
\hline & & 2013 & 2014 & 2015 & 2013 & 2014 & 2015 & $\begin{array}{l}p^{-} \\
\text {value } \\
\text { for } \\
\text { trend }\end{array}$ \\
\hline Total & & 1421 & 1209 & 1283 & & & & 0.035 \\
& & & & $N$ & MoT \% & MoT \% & MoT \% & \\
& HET & 868 & 725 & 808 & $61.1 \%$ & $60.0 \%$ & $63.0 \%$ & 0.329 \\
& IDU & 490 & 431 & 409 & $34.5 \%$ & $35.6 \%$ & $31.9 \%$ & 0.166 \\
& MSM & 47 & 47 & 61 & $3.3 \%$ & $3.9 \%$ & $4.8 \%$ & 0.055 \\
& OTH & 16 & 6 & 5 & $1.1 \%$ & $0.5 \%$ & $0.4 \%$ & 0.019 \\
\hline
\end{tabular}

Modes of transmission: HET heterosexual, IDU injecting drug use, MSM homosexual, OTH other
Table 7 Trends in modes of transmission by sex and age in the verified registry

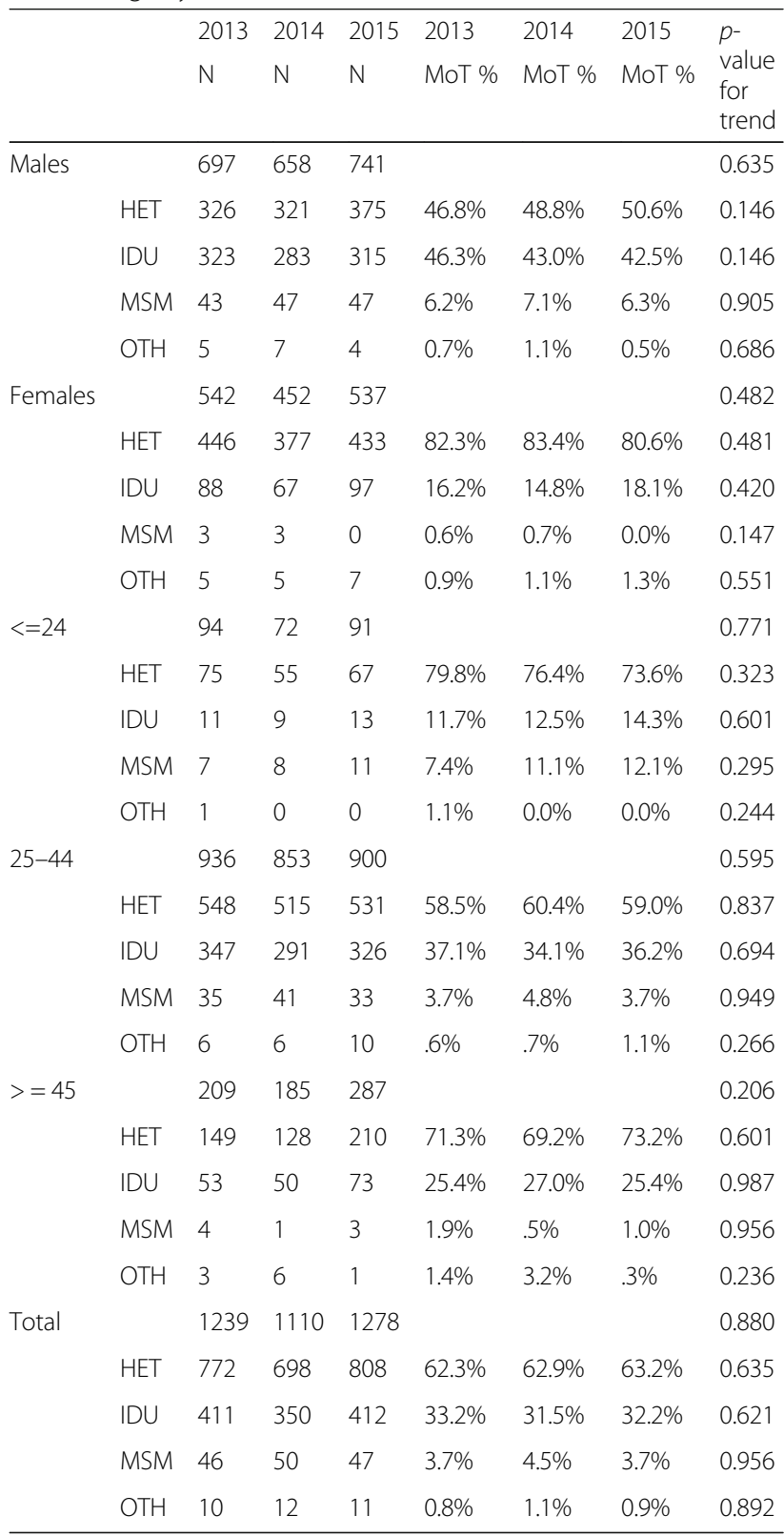

Modes of transmission: HET heterosexual, IDU injecting drug use, MSM homosexual, $\mathrm{OTH}$ other

increase was particularly obvious among men younger than 25 years: from $14.3 \%$ (2013) to $27.3 \%$ (2014) to 61.9\% (2015; $p=0.004)$. Nosocomial exposure increased from $10.8 \%$ (2013) to $18.5 \%$ (2014) to $17.8 \%$ (2015; $p<$ 0.001). HBV exposure and skin penetration factors did not change significantly in men or women.

\section{Accuracy of reporting}

As shown in Supplement Table S3, the distribution of the four registered modes of transmission in the official reports and in the verified registry did not significantly differ. With 
Table 8 Trends in modes of transmission by sex and age in the survey

\begin{tabular}{|c|c|c|c|c|c|c|c|c|}
\hline & & 2013 & 2014 & 2015 & 2013 & 2014 & 2015 & \\
\hline & & $N$ & $N$ & $N$ & МоТ \% & MoT \% & МоТ \% & $\begin{array}{l}\text { value } \\
\text { for } \\
\text { trend }\end{array}$ \\
\hline Males & & 409 & 369 & 468 & & & & 0.024 \\
\hline & HET & 94 & 82 & 111 & $23.0 \%$ & $22.2 \%$ & $23.7 \%$ & 0.784 \\
\hline & IDU & 296 & 263 & 314 & $72.4 \%$ & $71.3 \%$ & $67.1 \%$ & 0.085 \\
\hline & MSM & 19 & 24 & 43 & $4.6 \%$ & $6.5 \%$ & $9.2 \%$ & 0.008 \\
\hline & OTH & 0 & 0 & 0 & $0.0 \%$ & $0.0 \%$ & $0.0 \%$ & \\
\hline Females & & 357 & 312 & 363 & & & & 0.264 \\
\hline & HET & 168 & 173 & 197 & $47.1 \%$ & $55.4 \%$ & $54.3 \%$ & 0.054 \\
\hline & IDU & 188 & 136 & 164 & $52.7 \%$ & $43.6 \%$ & $45.2 \%$ & 0.045 \\
\hline & MSM & 0 & 0 & 0 & $0.0 \%$ & $0.0 \%$ & $0.0 \%$ & \\
\hline & OTH & 1 & 3 & 2 & $0.3 \%$ & $1.0 \%$ & $0.6 \%$ & 0.636 \\
\hline$<=24$ & & 51 & 45 & 56 & & & & 0.033 \\
\hline & HET & 39 & 38 & 37 & $76.5 \%$ & $84.4 \%$ & $66.1 \%$ & 0.923 \\
\hline & IDU & 10 & 4 & 6 & $19.6 \%$ & $8.9 \%$ & $10.7 \%$ & 0.025 \\
\hline & MSM & 2 & 3 & 13 & $3.9 \%$ & $6.7 \%$ & $23.2 \%$ & 0.002 \\
\hline & OTH & 0 & 0 & 0 & $0.0 \%$ & $0.0 \%$ & $0.0 \%$ & \\
\hline $25-44$ & & 583 & 523 & 594 & & & & 0.154 \\
\hline & HET & 272 & 254 & 286 & $46.7 \%$ & $48.6 \%$ & $48.1 \%$ & 0.200 \\
\hline & IDU & 296 & 251 & 277 & $50.8 \%$ & $48.0 \%$ & $46.6 \%$ & 0.059 \\
\hline & MSM & 14 & 18 & 31 & $2.4 \%$ & $3.4 \%$ & $5.2 \%$ & 0.058 \\
\hline & ОTH & 1 & 0 & 0 & $.2 \%$ & $0.0 \%$ & $0.0 \%$ & 0.225 \\
\hline$>=45$ & & 129 & 108 & 171 & & & & 0.497 \\
\hline & HET & 75 & 52 & 89 & $58.1 \%$ & $48.1 \%$ & $52.0 \%$ & 0.968 \\
\hline & IDU & 50 & 49 & 72 & $38.8 \%$ & $45.4 \%$ & $42.1 \%$ & 0.931 \\
\hline & MSM & 4 & 4 & 7 & $3.1 \%$ & $3.7 \%$ & $4.1 \%$ & 0.898 \\
\hline & OTH & 0 & 3 & 3 & $0.0 \%$ & $2.8 \%$ & $1.8 \%$ & 0.440 \\
\hline Total & & 766 & 681 & 831 & & & & 0.020 \\
\hline & HET & 262 & 255 & 308 & $34.2 \%$ & $37.4 \%$ & $37.1 \%$ & 0.242 \\
\hline & IDU & 484 & 399 & 478 & $63.2 \%$ & $58.6 \%$ & $57.5 \%$ & 0.022 \\
\hline & MSM & 19 & 24 & 43 & $2.5 \%$ & $3.5 \%$ & $5.2 \%$ & 0.005 \\
\hline & OTH & 1 & 3 & 2 & $0.1 \%$ & $0.4 \%$ & $0.2 \%$ & 0.686 \\
\hline
\end{tabular}

Modes of transmission: HET heterosexual, IDU injecting drug use, MSM homosexual, OTH other

the exception of Lviv, where not all registration forms were available for verification, the registered modes of transmission did not significantly differ at the regional level.

\section{Discussion}

In this study among patients registered with a diagnosis of HIV infection in 7 regions of Ukraine, we undertook a standardized ascertainment of risk factor information that was designed to be more sensitive through simple efforts to develop enhanced rapport and use of biological markers. Our results demonstrate that standard case registration procedures in HIV clinics in Ukraine are less sensitive in detecting stigmatized behaviors, such as homosexual exposure and injecting drug use, which leads to underestimation of the proportion of cases attributable to these modes of transmission. About two-thirds of patients who self-reported injecting drug use exposure and slightly less than half of those with $\mathrm{HCV}$ markers had injecting drug use as the registered mode of transmission. Only about half of men reporting sex with men (who did not inject drugs) had homosexual exposure as the registered mode of transmission. Consequently, the proportion of heterosexual transmission was overestimated by almost $75 \%$. Our findings suggest that Cakalo et al. [8] overestimated the proportion of MSM among men reported as heterosexual $(8.2 \%$ compared to $5.8 \%$ in our data, see Table 4) and underestimated the proportion of PWID (34.5\% compared to $54.3 \%$ in our data).

After adjusting for misclassification, the national distribution of homosexual exposure as a mode of transmission among HIV cases registered in Ukraine in 2015 was 4.2\%, which is close to the average of $4.0 \%$ in Eastern European countries [5]. The estimated $52.1 \%$ of transmission via injecting drug use in Ukraine remains higher than the average of $26 \%$ in the rest of Eastern Europe (excluding Russia, where injecting drug use still accounts for more than half of new HIV infections). Heterosexual transmission of HIV, therefore, is lower than in other Eastern European countries and, as other analyses show, in many cases occurs among partners of PWID [10]. Our findings suggest that the HIV epidemic in Ukraine remains significantly driven by injecting drug use.

We investigated the trends in HIV transmission in seven regions in Ukraine over 3 years (2013-2015). The prevalence of injecting drug use exposure remained high overall but significantly decreased among women and participants younger than 25 years. This finding may suggest a shift from injecting drug use to heterosexual transmission, which accounted for over half of cases among female participants in 2015.

The number and proportion of HIV cases attributable to homosexual exposure more than doubled between 2013 and 2015. Among men younger than 25 years, the proportion of cases attributable to homosexual exposure increased more than six times, from 14.3 to $61.9 \%$. This sharp increase largely occurred in two regions, Kyiv and Lviv, which may indicate an ongoing outbreak, especially among young MSM. Other evidence supports this possibility, including a high HIV incidence rate estimated from LAg assay testing of specimens from the 2013 MSM integrated bio-behavioral survey (IBBS) [19] and increased HIV prevalence in younger MSM according to the 2015 and 2013 IBBS surveys [12]. Importantly, this trend was not significant in the official reports and verified registration records owing to the substantial degree of misclassification. Given the relatively low proportion 
Table 9 Trends in risk factors by sex and age in the survey

\begin{tabular}{|c|c|c|c|c|c|c|c|c|}
\hline & & 2013 & 2014 & 2015 & 2013 & 2014 & 2015 & \\
\hline & & $\mathrm{N}$ & $\mathrm{N}$ & $N$ & $\%$ & $\%$ & $\%$ & $\begin{array}{l}\text { value } \\
\text { for } \\
\text { trend }\end{array}$ \\
\hline \multirow[t]{10}{*}{ Men } & & 410 & 373 & 470 & & & & \\
\hline & het & 401 & 360 & 442 & $97.8 \%$ & $96.5 \%$ & $94.0 \%$ & 0.004 \\
\hline & hrh & 251 & 213 & 274 & $61.4 \%$ & $57.4 \%$ & $58.4 \%$ & 0.476 \\
\hline & sti & 154 & 143 & 197 & $40.3 \%$ & $39.7 \%$ & $44.5 \%$ & 0.183 \\
\hline & idu & 246 & 226 & 263 & $60.0 \%$ & $60.9 \%$ & $56.3 \%$ & 0.264 \\
\hline & hcv & 250 & 232 & 273 & $61.4 \%$ & $65.4 \%$ & $59.3 \%$ & 0.595 \\
\hline & hbv & 206 & 183 & 239 & $50.6 \%$ & $51.5 \%$ & $52.1 \%$ & 0.579 \\
\hline & msm & 26 & 38 & 66 & $6.4 \%$ & $10.5 \%$ & $14.5 \%$ & $<0.001$ \\
\hline & nos & 29 & 58 & 67 & $7.4 \%$ & $15.8 \%$ & $14.7 \%$ & 0.002 \\
\hline & pen & 262 & 231 & 296 & $70.1 \%$ & $67.3 \%$ & $68.7 \%$ & 0.787 \\
\hline \multirow[t]{10}{*}{ Women } & & 357 & 312 & 363 & & & & \\
\hline & het & 356 & 306 & 354 & $99.7 \%$ & $98.1 \%$ & $97.5 \%$ & 0.016 \\
\hline & hrh & 174 & 145 & 144 & $48.7 \%$ & $46.9 \%$ & $39.8 \%$ & 0.020 \\
\hline & sti & 122 & 106 & 146 & $37.5 \%$ & $35.1 \%$ & $42.8 \%$ & 0.130 \\
\hline & idu & 110 & 78 & 92 & $31.1 \%$ & $25.2 \%$ & $25.8 \%$ & 0.119 \\
\hline & hcv & 167 & 122 & 137 & $47.4 \%$ & $41.8 \%$ & $38.1 \%$ & 0.023 \\
\hline & hbv & 147 & 110 & 129 & $41.8 \%$ & $37.7 \%$ & $35.8 \%$ & 0.151 \\
\hline & $\mathrm{msm}$ & 0 & 0 & 0 & $0.0 \%$ & $0.0 \%$ & $0.0 \%$ & \\
\hline & nos & 51 & 66 & 77 & $14.7 \%$ & $21.7 \%$ & $21.9 \%$ & 0.022 \\
\hline & pen & 198 & 153 & 198 & $60.0 \%$ & $53.3 \%$ & $58.1 \%$ & 0.812 \\
\hline \multirow[t]{10}{*}{$<=24$} & & 52 & 46 & 59 & & & & \\
\hline & het & 52 & 45 & 53 & $100.0 \%$ & $97.8 \%$ & $89.8 \%$ & 0.008 \\
\hline & hrh & 26 & 11 & 16 & $50.0 \%$ & $23.9 \%$ & $27.6 \%$ & 0.029 \\
\hline & sti & 14 & 13 & 19 & $31.8 \%$ & $30.2 \%$ & $35.2 \%$ & 0.562 \\
\hline & idu & 10 & 4 & 6 & $19.6 \%$ & $8.9 \%$ & $10.5 \%$ & 0.184 \\
\hline & hcv & 20 & 11 & 10 & $38.5 \%$ & $24.4 \%$ & $16.9 \%$ & 0.024 \\
\hline & hbv & 17 & 11 & 19 & $32.7 \%$ & $24.4 \%$ & $32.2 \%$ & 0.757 \\
\hline & msm & 2 & 3 & 13 & $14.3 \%$ & $27.3 \%$ & $61.9 \%$ & 0.004 \\
\hline & nos & 2 & 11 & 9 & $4.0 \%$ & $25.0 \%$ & $16.1 \%$ & 0.119 \\
\hline & pen & 25 & 16 & 35 & $51.0 \%$ & $38.1 \%$ & $62.5 \%$ & 0.141 \\
\hline \multirow[t]{10}{*}{$25-44$} & & 585 & 528 & 599 & & & & \\
\hline & het & 578 & 518 & 584 & $98.8 \%$ & $98.1 \%$ & $97.5 \%$ & 0.089 \\
\hline & hrh & 338 & 293 & 327 & $57.9 \%$ & $55.6 \%$ & $54.7 \%$ & 0.312 \\
\hline & sti & 209 & 193 & 244 & $38.5 \%$ & $37.7 \%$ & $43.3 \%$ & 0.090 \\
\hline & idu & 296 & 251 & 277 & $50.7 \%$ & $47.8 \%$ & $46.6 \%$ & 0.156 \\
\hline & hcv & 335 & 289 & 312 & $58.0 \%$ & $58.0 \%$ & $52.9 \%$ & 0.097 \\
\hline & hbv & 273 & 227 & 269 & $47.2 \%$ & $45.6 \%$ & $45.7 \%$ & 0.693 \\
\hline & $\mathrm{msm}$ & 20 & 29 & 44 & $6.3 \%$ & $10.1 \%$ & $12.6 \%$ & 0.006 \\
\hline & nos & 55 & 88 & 92 & $9.8 \%$ & $17.0 \%$ & $15.8 \%$ & 0.004 \\
\hline & pen & 364 & 310 & 356 & $67.8 \%$ & $63.4 \%$ & $64.7 \%$ & 0.386 \\
\hline \multirow[t]{2}{*}{$>=45$} & & 130 & 111 & 175 & & & & \\
\hline & het & 127 & 103 & 159 & $97.7 \%$ & $92.8 \%$ & $90.9 \%$ & 0.019 \\
\hline
\end{tabular}


Table 9 Trends in risk factors by sex and age in the survey (Continued)

\begin{tabular}{|c|c|c|c|c|c|c|c|c|}
\hline & & 2013 & 2014 & 2015 & 2013 & 2014 & 2015 & \\
\hline & & $N$ & $N$ & $N$ & $\%$ & $\%$ & $\%$ & $\begin{array}{l}\text { value } \\
\text { for } \\
\text { trend }\end{array}$ \\
\hline & hrh & 61 & 54 & 75 & $46.9 \%$ & $50.5 \%$ & $42.9 \%$ & 0.442 \\
\hline & sti & 53 & 43 & 80 & $44.2 \%$ & $40.2 \%$ & $48.2 \%$ & 0.412 \\
\hline & idu & 50 & 49 & 72 & $38.8 \%$ & $44.1 \%$ & $41.9 \%$ & 0.605 \\
\hline & hcv & 62 & 54 & 88 & $48.1 \%$ & $51.9 \%$ & $51.5 \%$ & 0.412 \\
\hline & hbv & 63 & 55 & 80 & $48.8 \%$ & $52.9 \%$ & $46.8 \%$ & 0.690 \\
\hline & $\mathrm{msm}$ & 4 & 6 & 9 & $5.4 \%$ & $9.2 \%$ & $10.3 \%$ & 0.364 \\
\hline & nos & 23 & 25 & 43 & $18.3 \%$ & $22.5 \%$ & $25.3 \%$ & 0.157 \\
\hline & pen & 71 & 58 & 103 & $60.2 \%$ & $58.6 \%$ & $62.0 \%$ & 0.642 \\
\hline Total & & 767 & 685 & 833 & & & & \\
\hline & het & 757 & 666 & 796 & $98.7 \%$ & $97.2 \%$ & $95.6 \%$ & $<0.001$ \\
\hline & hrh & 425 & 358 & 418 & $55.5 \%$ & $52.6 \%$ & $50.3 \%$ & 0.057 \\
\hline & sti & 276 & 249 & 343 & $39.0 \%$ & $37.6 \%$ & $43.8 \%$ & 0.042 \\
\hline & idu & 356 & 304 & 355 & $46.6 \%$ & $44.6 \%$ & $43.1 \%$ & 0.172 \\
\hline & hcv & 417 & 354 & 410 & $54.9 \%$ & $54.7 \%$ & $50.0 \%$ & 0.092 \\
\hline & hbv & 353 & 293 & 368 & $46.5 \%$ & $45.3 \%$ & $44.9 \%$ & 0.692 \\
\hline & $\mathrm{msm}$ & 26 & 38 & 66 & $6.4 \%$ & $10.5 \%$ & $14.5 \%$ & $<0.001$ \\
\hline & nos & 80 & 124 & 144 & $10.8 \%$ & $18.5 \%$ & $17.8 \%$ & $<0.001$ \\
\hline & pen & 460 & 384 & 494 & $65.3 \%$ & $61.0 \%$ & $64.0 \%$ & 0.819 \\
\hline
\end{tabular}

Risk factors (for definitions see Table 1): het heterosexual exposure, $h r h$ high-risk heterosexual exposure, sti sexually transmitted infections, idu injecting drug use, $h c v$ exposure to $\mathrm{HCV}, h b v$ exposure to $\mathrm{HBV}, \mathrm{msm}$ homosexual exposure, nos nosocomial exposure, pen skin penetration exposure

of MSM cases overall, misclassification may obscure potential outbreaks and delay the public health response.

\section{Limitations}

Our study has several limitations. First, we relied on self-report of risk behaviors, which is prone to recall bias and deliberate underreporting of stigmatized behaviors. To mitigate this limitation, we tested for biological markers and assumed 100\% link between the positive anti-HCV results and injecting drug use. On the other hand, prevalence of $\mathrm{HCV}$ in the general population in Ukraine may be substantial [20], which may have led to

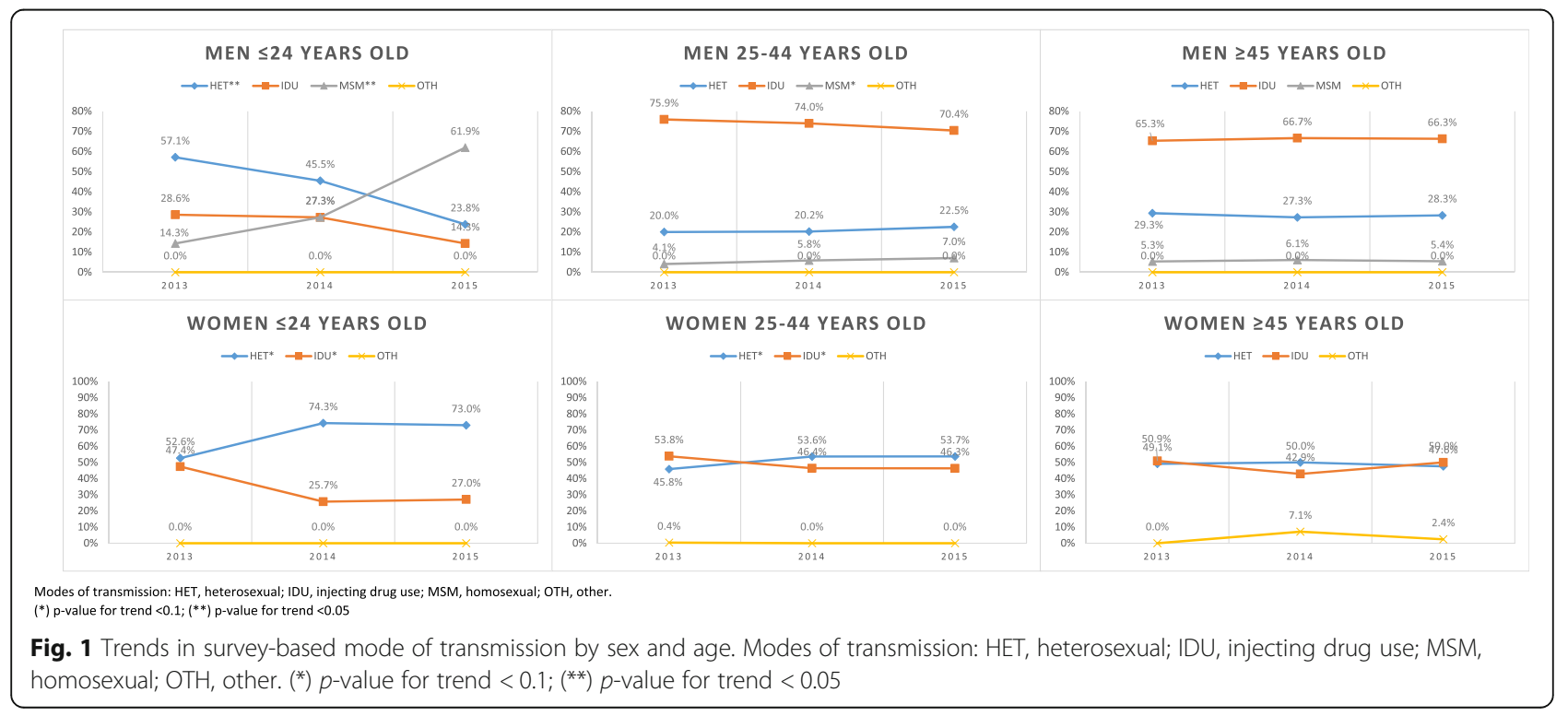


overestimation of the number of injecting drug userelated cases in our study.

Another important limitation of self-reported HIV risk is the uncertainty about the specific behavior that actually led to transmission. Nearly all participants reported heterosexual activity, and about two-thirds reported skin penetration exposure. Thus, there is a possibility that homosexual exposure or injecting drug use were not the actual causes of HIV transmission in these patients. We suggested a hierarchy of most probable modes of transmission based on the probability of transmission per act [16] and the prevalence of infection in respective populations in Ukraine [21, 22], This hierarchy, we believe, has the least bias in the current epidemiological context. A study of risk networks with virus genotyping is needed to establish the probability of acquiring HIV through specific modes when multiple exposures are present.

In our study sample, the number of patients with homosexual exposure as the registered mode of transmission was disproportionately lower than among registered patients who did not participate in our study; therefore the observed prevalence of homosexual exposure in our sample likely underestimates the true level. However, this should not bias our estimate of the degree of misclassification and hence the estimated proportion of HIV infections attributable to homosexual exposure among all patients nationally.

\section{Conclusion}

There is a significant degree of misclassification of key modes of transmission in the case registration system in Ukraine. Improvements in HIV case registration systems, such as more structured and sensitive ascertainment of risk factors, are needed to more accurately assess the epidemic trends and guide programmatic response in Ukraine and other countries where injecting drug use and homosexuality are stigmatized.

We found that HIV transmission via injecting drug use is still high, particularly among men, although this mode of transmission is decreasing significantly. We also found an explosive increase, more than double overall and more than six times in patients younger than 25 years in only 2 years, of HIV infections attributed to homosexual exposure, which correlates with other data $[12,19]$. In a resource-limited context, both key populations would benefit from combination prevention, including traditional harm reduction and medicationassisted treatment for opioid users as well as novel interventions such as pre-exposure prophylaxis and the Test and Treat approach. Targeting these key populations (MSM and PWID) can help Ukraine achieve ambitious targets set by the World Health Organization European Member States in September 2016 [23] and prevent outbreaks that may occur due to reduction of harm reduction activities [24-28].

\section{Supplementary information}

Supplementary information accompanies this paper at https://doi.org/10. 1186/s12889-020-09373-2.

Additional file 1: Supplementary file 1. Participant questionnaire. This is the original version of the study questionnaire in Ukrainian with machine-translated English version.

Additional file 2: Table S1. Site selection. Table S2. Study population and sample. Table S3. Distribution of registered modes of transmission in the official reports, verified registry and study sample. Table S4. Prevalence of risk factors by registered mode of transmission and region. Table S5. Misclassification of modes of transmission by region. Table S6. Trends in modes of transmission by region in the official reports. Table S7. Trends in modes of transmission by region in the verified registry. Table S8. Trends in modes of transmission by region in the survey. Table S9. Trends in risk factors by region in the survey.

\section{Abbreviations}

AIDS: Acquired immunodeficiency syndrome; CDC : US Centers for disease control and prevention; HBV: Hepatitis B virus; HCV: Hepatitis C virus; HIV: Human immunodeficiency virus; MSM: Men who have sex with men; PWID: People who inject drugs

\section{Acknowledgments}

The authors thank Maria Samko, Liudmila Khomych, Ivana Bozicevic, JurjaIvana Čakalo and Natalia Saichuk for their contribution to the study design and data collection.

\section{Authors' contributions}

C.V., M.A., and K.D. conceptualized and designed the study. R.K., C.V., and M.K. contributed to the analysis approach. K.D. managed and analyzed the data. K.D. and M.K. wrote the paper. All authors have read and approved the final manuscript.

\section{Funding}

This project has been supported by the U.S. President's Emergency Plan for AIDS Relief (PEPFAR) through the U.S. Centers for Disease Control and Prevention under the terms of grant NU2GGH000840 (Engaging Local Indigenous Organizations in Developing HIV/AIDS Monitoring and Evaluation Capacity in Ukraine). The findings and conclusions in this report are those of the authors and do not necessarily represent the official position of the funding agencies.

\section{Availability of data and materials}

The de-personalized datasets used in the current study are available from the corresponding author on reasonable request. Summary tables with sitelevel data are presented as Supplements. The study questionnaire is provided in Supplementary Files.

\section{Ethics approval and consent to participate}

The study protocol was approved by the Ukrainian Institute on Public Health Policy Institutional Review Board \#1 in August 2015. The study was reviewed in accordance with the U.S. Centers for Disease Control and Prevention (CDC) human research protection procedures and determined to be research, but CDC investigators did not interact with human subjects or have access to identifiable data or specimens for research purposes. All participants provided written informed consent.

\section{Consent for publication}

Not applicable.

\section{Competing interests}

The authors declare that they have no competing interests.

\section{Author details}

${ }^{1}$ Ukrainian Institute on Public Health Policy, Kyiv, Ukraine. ${ }^{2}$ Alliance for Public Health, Kyiv, Ukraine. ${ }^{3}$ Division of Global HIV and TB, Center for Global Health, U.S. Centers for Disease Control and Prevention, Kyiv, Ukraine. ${ }^{4}$ Division of Global HIV and TB, Center for Global Health, U.S. Centers for Disease Control and Prevention, Atlanta, GA, USA. 
Received: 6 November 2019 Accepted: 11 August 2020

Published online: 26 August 2020

\section{References}

1. Wilson D, Halperin DT. "know your epidemic, know your response": a useful approach, if we get it right. Lancet. 2008;372(9637):423-6.

2. Zimmerman RS, Morisky DE, Harrison L, Mark HD. Validity of behavioral measures as proxies for HIV-related outcomes. J Acquir Immune Defic Syndr. 2014;66(Suppl 3(0 3)):S285-92.

3. Palamar JJ, Le A, Guarino H, Mateu-Gelabert P. A comparison of the utility of urine- and hair testing in detecting self-reported drug use among young adult opioid users. Drug Alcohol Depend. 2019;200:161-7.

4. Centers for Disease Control and Prevention. HIV Surveillance Report, vol. 26; 2014. Available from: https://www.cdc.gov/hiv/pdf/library/reports/ surveillance/cdc-hiv-surveillance-report-us.pdf.

5. European Centre for Disease Prevention and Control, WHO Regional Office for Europe. HIV/AIDS surveillance in Europe 2019-2018 data. Stockholm: ECDC; 2019. Available from: https://www.ecdc.europa.eu/en/publicationsdata/hivaids-surveillance-europe-2019-2018-data.

6. Demchenko I, Dmitriev D. Measurement of stigma toward PLWH: comparative analysis of stigma index surveys 2010, 2013 and 2016: AllUkrainian Network of PLWH; 2016. Available from: https://issuu.com/ network_of_plwh/docs/presentstion_stigma_index_2016_resu.

7. Harrison KM, Kajese T, Hall HI, Song R. Risk factor redistribution of the national HIV/AIDS surveillance data: an alternative approach. Public Health Rep. 2008:123(5):618-27.

8. Cakalo Jl, Bozicevic I, Vitek C, Mandel JS, Salyuk T, Rutherford GW. Misclassification of men with reported HIV infection in Ukraine. AIDS Behav. 2015;19(10):1938-40.

9. Spindler H, Salyuk T, Vitek C, Rutherford G. Underreporting of HIV transmission among men who have sex with men in the Ukraine. AIDS Res Hum Retrovir. 2014;30(5):407-8.

10. Vitek CR, Cakalo Jl, Kruglov Y, Dumchev KV, Salyuk TO, Bozicevic I, et al. Slowing of the HIV epidemic in Ukraine: evidence from case reporting and key population surveys, 2005-2012. PLoS One. 2014;9(9):e103657.

11. MoH of Ukraine. HIV infection in Ukraine Informational Bulletin \#45. Kyiv: Ministry of Health of Ukraine, Ukrainian Center for Socially Dangerous Diseases Control, Gromashevsky Institute of Epidemiology and Infectious Diseases; 2016. Available from: http://phc.org.ua/pages/diseases/hiv_aids/ monitoring/information-bulletins.

12. Dumchev K, Sazonova Y, Salyuk T, Varetska O. Trends in HIV prevalence among people injecting drugs, men having sex with men, and female sex workers in Ukraine. Int J STD AIDS. 2018;29(13):1337-44.

13. MoH of Ukraine. HIV infection in Ukraine Informational Bulletin \#43. Kyiv: Ministry of Health of Ukraine, Ukrainian Center for Socially Dangerous Diseases Control, Gromashevsky Institute of Epidemiology and Infectious Diseases; 2015. Available from: https://www.phc.org.ua/kontrolzakhvoryuvan/vilsnid/monitoring-i-ocinka/informaciyni-byuleteni-vilsnid.

14. Harris PA, Taylor R, Thielke R, Payne J, Gonzalez N, Conde JG. Research electronic data capture (REDCap)--a metadata-driven methodology and workflow process for providing translational research informatics support. $J$ Biomed Inform. 2009;42(2):377-81.

15. Hall HI, Song R, Tang T, An Q, Prejean J, Dietz P, et al. HIV trends in the United States: diagnoses and estimated incidence. JMIR Public Health Surveill. 2017;3(1):e8.

16. Patel P, Borkowf CB, Brooks JT, Lasry A, Lansky A, Mermin J. Estimating peract HIV transmission risk: a systematic review. Aids. 2014;28(10):1509-19.

17. Lee LM, McKenna MT, Janssen RS. Classification of transmission risk in the national HIV/AIDS surveillance system. Public Health Rep. 2003;118(5):400-7.

18. Stokes ME, Davis CS, Koch GG. Categorical data analysis using the SAS system. 2nd ed. Cary: SAS Institute; 2000. viii, 626 p. p.

19. Public Health Center of the MoH of Ukraine, Gromashevsky Institute of Epidemiology and Infectious Diseases. HIV infection in Ukraine Informational Bulletin \#49 Kyiv, 2018. p. 42-3. Available from: https://www.phc.org.ua/ kontrol-zakhvoryuvan/vilsnid/monitoring-i-ocinka/informaciyni-byuletenivilsnid.

20. Hope VD, Eramova I, Capurro D, Donoghoe MC. Prevalence and estimation of hepatitis $B$ and $C$ infections in the WHO European region: a review of data focusing on the countries outside the European Union and the European free trade association. Epidemiol Infect. 2014;142(2):270-86.
21. Barska J, Sazonova YO. Monitoring of behaviour and HIV prevalence among people who inject drugs and their sexual partners: analytical report on 2015 study. Kyiv: Alliance for Public Health; 2016. Available from: http://aph.org. ua/wp-content/uploads/2015/09/monsin.pdf.

22. Kasianczuk M, Trofymenko O, Bilous Y, Sazonova YO. Monitoring of behaviour and HIV prevalence among men who have sex with men: analytical report on 2015 study. Kyiv: Alliance for Public Health; 2017. Available from: http://aph.org.ua/wp-content/uploads/2017/06/ Analituchnuy_zvit_Nacionalna-chastyna_28.06.2017_com.pdf.

23. The WHO Regional Office for Europe. Action plan for the health sector response to HIV in the WHO European Region 2017. Available from: http:// www.euro.who.int/en/media-centre/events/events/2018/04/hiv-in-europe-andcentral-asia-in-the-era-of-the-sdgs-operationalizing-goals-and-achieving-targets.

24. National Services Scotland. HPS weekly report Glasgow (Clifton House, Clifton Place, Glasgow G3 7LN): HPS; 2016. updated Volume 50 No. 2016/11. Available from: http://www.hps.scot.nhs.uk/documents/ewr/pdf2016/1611.pdf.

25. Botescu A, Abagiu A, Mardarescu M, Ursan M. HIV/AIDS among injecting drug users in Romania. In: Report of a recent outbreak and initial response policies. Lisbon: EMCDDA; 2012. updated Lisbon: EMCDDA. Available from: http://www. emcdda.europa.eu/publications/ad-hoc/2012/romania-hiv-update.

26. Tarjan A, Dudas M, Wiessing L, Horvath G, Rusvai E, Treso B, et al. HCV prevalence and risk behaviours among injectors of new psychoactive substances in a risk environment in Hungary-An expanding public health burden. Int J Drug Policy. 2017;41:1-7.

27. Fotiou A, Micha K, Paraskevis D, Terzidou M, Malliori M, Hatzakis A. HIV outbreak among injecting drug users in Greece. An Updated brief report for the EMCDDA on the recent outbreak of HIV infections among drug injectors in Greece. Lisbon: European Monitoring Centre for Drugs and Drug Addiction; 2012. updated Lisbon: EMCDDA. Available from: http://www. emcdda.europa.eu/publications/ad-hoc/2012/greece-hiv-update.

28. Des Jarlais DC, Kerr T, Carrieri P, Feelemyer J, Arasteh K. HIV infection among persons who inject drugs: ending old epidemics and addressing new outbreaks. Aids. 2016:30(6):815-26.

\section{Publisher's Note}

Springer Nature remains neutral with regard to jurisdictional claims in published maps and institutional affiliations.
Ready to submit your research? Choose BMC and benefit from:

- fast, convenient online submission

- thorough peer review by experienced researchers in your field

- rapid publication on acceptance

- support for research data, including large and complex data types

- gold Open Access which fosters wider collaboration and increased citations

- maximum visibility for your research: over $100 \mathrm{M}$ website views per year

At $\mathrm{BMC}$, research is always in progress.

Learn more biomedcentral.com/submissions 